\title{
THE PERIODOGRAM OF FRACTIONAL PROCESSES ${ }^{1}$
}

\author{
By Carlos Velasco \\ Universidad Carlos III de Madrid
}

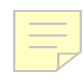

First Version received December 2004

\begin{abstract}
We analyse asymptotic properties of the discrete Fourier transform and the periodogram of time series obtained through (truncated) linear filtering of stationary processes. The class of filters contains the fractional differencing operator and its coefficients decay at an algebraic rate, implying long-range-dependent properties for the filtered processes when the degree of integration $\alpha$ is positive. These include fractional time series which are nonstationary for any value of the memory parameter $(\alpha \neq 0)$ and possibly nonstationary trending $(\alpha \geq 0.5)$. We consider both fractional differencing or integration of weakly dependent and long-memory stationary time series. The results obtained for the moments of the Fourier transform and the periodogram at Fourier frequencies in a degenerating band around the origin are weaker compared with the stationary nontruncated case for $\alpha>0$, but sufficient for the analysis of parametric and semiparametric memory estimates. They are applied to the study of the properties of the log-periodogram regression estimate of the memory parameter $\alpha$ for Gaussian processes, for which asymptotic normality could not be showed using previous results. However, only consistency can be showed for the trending cases, $0.5 \leq \alpha<1$. Several detrending and initialization mechanisms are studied and only local conditions on spectral densities of stationary input series and transfer functions of filters are assumed.
\end{abstract}

Keywords. Discrete Fourier transform; long-range dependence; long memory; nonstationary series; log-periodogram regression; asymptotic normality.

MSC. Primary: 62M15; Secondary: 62M10, 60G18.

\section{INTRODUCTION}

Most models for nonstationary trending and long memory time series are based on some underlying weakly dependent stationary processes. Thus for stationary long memory or long range dependent processes it is often assumed that

$$
x_{t} \quad(1 \quad L)^{-d} \varepsilon_{t}, \quad 0<d<0.5, \quad t \quad 1,2, \ldots,
$$

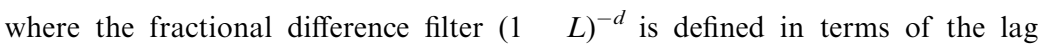
operator $L$ through the formal expansion,

$$
\left(\begin{array}{ll}
1 & L
\end{array}\right)^{-d}: \sum_{j}^{\infty} \varphi_{j}^{(d)} L^{j}, \quad \varphi_{j}^{(d)} \frac{\Gamma(j+d)}{\Gamma(d) \Gamma(j+1)},
$$

for any real $d / 1, \quad 2, \ldots$, where

$$
\Gamma(x) \quad \int_{0}^{\infty} z^{x-1} \mathrm{e}^{-z} \mathrm{~d} z
$$


is the Gamma function and $\Gamma(0) / \Gamma(0) \quad 1$. Here $\varepsilon_{t}, t \quad 0, \pm 1, \ldots$, is assumed to be covariance stationary and weakly dependent according to some standard definition, which usually entails that the process $\varepsilon_{t}$ has a positive bounded spectral density at zero frequency. For instance, Hosking (1981) and Granger and Joyeux (1980) originally proposed that $\varepsilon_{t}$ is an auto regressive moving average process. Then $x_{t}$ is termed integrated of order $d$, is covariance stationary with finite variance under $d<0.5$, and its spectral density has a pole (zero) at zero frequency if $d>0(d<0)$. Trending observations can be modelled by partial sums (Sowell, 1990; Hurvich and Ray, 1995; Velasco, 1999),

$$
y_{t} \quad y_{0}+\sum_{j=1}^{t} x_{j}, \quad t \quad 1,2, \ldots,
$$

leading to difference stationary models where the increment series $\left(\begin{array}{lll}1 & L\end{array}\right) y_{t} \quad x_{t}$ is stationary integrated of order $d \quad 1, \quad 0.5 \leq d \quad 1<0.5$, satisfying eqn (1). Then we say that $y_{t}$ is integrated of order $d, 0.5 \leq d<1.5$. Using successive partial sums it is straightforward to define higher order integrated series for any $d \geq 0.5$.

Alternatively, it is possible to consider (e.g. Phillips, 1999; Robinson and Marinucci, 2001) that a process is generated by a truncated filtering as

$$
\zeta_{t} \quad \sum_{j}^{t-1} \varphi_{j}^{(\alpha)} \eta_{t-j}, \quad \alpha>0, \quad t \quad 1,2, \ldots,
$$

so all the past weakly dependent stationary innovations $\eta_{t}, t \leq 0$, are ignored. This convention makes essential the date of start of observation. However, this framework can easily be generalized setting a warming up period where the inflow of information can begin before we actually observe the process. The filtered process $\zeta_{t}$, although with finite variance for fixed $t$, is nonstationary for any value of $\alpha / 0$. However, if $\alpha<0.5$, it converges in mean square as $t \rightarrow \infty$ to the covariance stationary $x_{t}$ obtained in eqn (1) for the same sequence of innovations $\eta_{j} \quad \varepsilon_{j}, j \quad 1, \ldots, t$. Using Stirling's formula, we found that $\Gamma(\alpha) \varphi_{j}^{(\alpha)} \sim j^{\alpha-1}$ as $j \rightarrow \infty$, where $\sim$ means that the ratio of left and right hand sides tends to 1 . Therefore, when $\alpha \geq 0.5$ the variance of $\zeta_{t}$ can grow without limit with $t$ and $\zeta_{t}$ is nonstationary long range dependent in the sense of Heyde and Yang (1997). Of course, both ideas can be combined to define fractional integrated processes, achieving complete generality in the generation of time series with long range dependent behaviour.

The long range properties of the processes (1) (3) are described by the memory parameters $d$ and $\alpha$, and under regularity conditions and appropriately normalized, such processes converge to different versions of fractional Brownian motion of parameters $d>0.5$ and $\alpha>0.5$ respectively (see Marinucci and Robinson, 2000 for a discussion). The memory parameters can be estimated by a variety of methods under general assumptions. Main focus in the literature has been paid to stationary long memory series (e.g. Fox and 
Taqqu, 1986; Robinson, 1995a,b), although difference stationary models have been considered (e.g. Velasco, 1999; Velasco and Robinson, 2000). Using the other alternative, Phillips (1999) considered asymptotic properties of the discrete Fourier transform (DFT) of $\zeta_{t}$ given by eqn (3), where $\eta_{t}$ is a linear process with coefficients in the Wold decomposition associated with a uniformly smooth spectral density, extending ideas of Phillips and Solo (1992) in terms of a Barlett's approximation to the DFT of the linear i.i.d. innovations. Robinson (2005) considered bounds between the DFT of $\zeta_{t}$ and its stationary version $x_{t}$ given by eqn (1) when $\alpha \quad d \in[0.5,0.5)$ and $\varepsilon_{t} \quad \eta_{t}$, and studied the effect of tapering when these series are possibly further fractionally integrated to achieve nonstationary memory levels. These results are used to derive the properties of different parameter estimates which are linear functions of the periodogram, and whose analysis had been conducted previously for (difference) stationary processes.

In this paper, we generalize and improve the previous results on the moments of DFT and periodogram for frequency domain inference on fractional series given by eqn (3) following a different route. In accordance with Robinson (1995a), we study the DFT of the observed series directly and set our local conditions in terms of the spectral density of the weakly dependent innovations $\eta_{t}$ and of a general nonstationary long range dependent filter as in Robinson and Marinucci (2001). In this paper, we also consider several extensions of model (3). We consider simultaneously series with negative memory $(\alpha<0)$, which are relevant for statistical inference on fractionally differenced data; processes with filters initialized at a point different from zero; and fractional differencing and integration of stationary long memory time series with $\eta_{t} \quad x_{t}$ given, e.g. by eqn (1) with $0<|d|<0.5$.

Our results are weaker than the ones that can be obtained in the nontruncated case for both $|\alpha|<0.5$ and $\alpha \geq 0.5$ (cf. Robinson, 1995a and Velasco, 1999, respectively). However, they are sufficient to justify valid large sample log periodogram inference on $\alpha,|\alpha|<0.5$ (Geweke and Porter Hudak, 1983; Robinson, 1995a) for Gaussian processes, although we can only obtain consistency results when $0.5 \leq \alpha<1$. The results allow for stationary long memory innovations, log periodogram regression estimating the overall memory of the resulting time series in this case. Previous results in Phillips (1999) and Robinson (2005) could not justify such asymptotic properties. Our local conditions on the spectral density of $\eta_{t}$ are also weaker and more general than those of Kim and Phillips (1999) who studied this semiparametric estimate when $\alpha \geq 1$ in a related set up following Phillips' (1999) analysis. The results obtained in this paper are also useful for other problems and, for instance, the narrow band Whittle estimate investigated in Robinson (1995b) for stationary long memory linear processes has been analysed in Marmol and Velasco (2004) under eqn (3) for linear $\eta_{t}$ and $|\alpha|<0.5$.

The rest of the paper is organized as follows. Next section analyses the covariance properties of the DFT of fractionally integrated series, while Section 3 considers the log periodogram estimate of $\alpha$. Section 4 discusses the initialization 
of the process $\zeta_{t}$ and different detrending mechanisms. The case when $\eta_{t}$ is a long memory (stationary) process is studied in Section 5. Some technical lemmas and proofs are contained in an appendix.

\section{DISCRETE FOURIER TRANSFORM OF FRACTIONALLY INTEGRATED TIME SERIES}

We follow the same notation as in Robinson and Marinucci (2001) but in a univariate framework to simplify the presentation. We consider linear filters more general than the fractional $(1 L)^{\alpha}$, with similar first order asymptotic properties. We consider simultaneously the cases of positive and negative memory parameter. The filter with positive memory parameter induces positive autocorrelation for long lags and is the relevant case for most data in practice, although negative values are likely to occur if some detrending mechanism, such as differencing, has been applied previously.

Generalizing eqn (3), which is stated in terms of the fractional weights $\varphi_{j}^{(\alpha)}$, we suppose that the observed sequence is given by

$$
\zeta_{t} \quad \sum_{j 1}^{t} \phi_{t-j}^{(\alpha)} \eta_{j}, \quad t \quad 1, \ldots, n,
$$

where the filter coefficients $\phi_{t}^{(\alpha)}$, its transfer function

$$
\phi(\lambda) \quad \sum_{j}^{\infty} \phi_{j}^{(\alpha)} \exp (\mathrm{i} j \lambda),
$$

and the process $\eta_{t}$ satisfy Assumption 1 .

Assumption 1.

1. $\left\{\phi_{t}^{(\alpha)}\right\} \in \Phi(\alpha)$, where $\Phi(\alpha),|\alpha|<1$, is the class of sequences $\left\{\phi_{t}^{(\alpha)}, t \quad 0,1, \ldots\right\}$, such that $\phi_{t}^{(0)} \quad 1(t \quad 0)$, and

$$
\begin{aligned}
\phi_{t}^{(\alpha)} & \sim \frac{t^{\alpha-1}}{\Gamma(\alpha)}, \quad \text { as } t \rightarrow \infty ; \\
\left|\phi_{t}^{(\alpha)} \quad \phi_{t+1}^{(\alpha)}\right| & O\left(t^{-1}\left|\phi_{t}^{(\alpha)}\right|\right), \quad \text { as } t \rightarrow \infty .
\end{aligned}
$$

2. $\phi(0) \quad \sum_{j 0}^{\infty} \phi_{j}^{(\alpha)} \quad 0$, and $|\phi(\lambda)| \sim|\lambda|^{-2 \alpha}$ as $|\lambda| \rightarrow 0, \quad 1<\alpha<0$.

3. $\eta_{t}$ is a zero mean covariance stationary process with spectral density $f_{\eta \eta}(\lambda)$, positive and continuous in an interval around $\lambda \quad 0$.

It is easy to show that the sequence $\varphi_{t}^{(\alpha)}$ given by the fractional filter $(1 L)^{-\alpha}$ belongs to the class $\Phi(\alpha)$, although our setup will not cover models such as eqn (1), defined in terms of the infinite sequence $\varphi_{j}^{(\alpha)}$. In fact, the class $\Phi(\alpha)$ is more general than that defined in terms of the fractional integration filter since we allow 
for contributions of a smaller order of integration $\alpha \quad \gamma, \gamma>0$, which can be of interest in some applications regarding cointegrated time series and volatility sequences (see e.g. the survey in Velasco, 2006). This possibility will be effectively accounted for in (2.) of Assumption 3. For $\alpha<0$, the class $\Phi(\alpha)$ could be defined in terms of differences of filters belonging to $\Phi(\alpha+1)$. Thus

$$
\phi_{t}^{(\alpha)} \quad \phi_{t}^{(\alpha+1)} \quad \phi_{t-1}^{(\alpha+1)}, \quad t \quad 1,2, \ldots, \quad \phi_{0}^{(\alpha)} \quad 1,
$$

as when we take fractional differences of a finite length vector observed in $t$ $1, \ldots, n$. For $\alpha>0$, the asymptotic behaviour of $|\phi(\lambda)|$ when $|\lambda| \rightarrow 0$, is deduced in Lemma 1 in the Appendix, using (1) of Assumption 1, and described by (2) of Assumption 1 for $1<\alpha<0$.

The conditions on $\eta_{t}$ imposed by (3) of Assumption 1 are not restrictive as we leave all the spectral density $f_{\eta \eta}(\lambda)$ unparametrized except at zero frequency, where it is smooth, and require only integrability for covariance stationarity. We further relax this condition in Section 5 and consider stationary long range dependent $\eta_{t}$ whose spectral density has singularities at $\lambda \quad 0$.

We are mainly concerned with the asymptotic properties of the DFT of $\zeta_{t}, t$ $1, \ldots, n$,

$$
w_{\zeta}\left(\lambda_{j}\right) \quad(2 \pi n)^{-1 / 2} \sum_{t}^{n} \zeta_{t} \exp \left(\mathrm{i} t \lambda_{j}\right)
$$

at the set of Fourier frequencies $\lambda_{j} 2 \pi j / n$, in a degenerating band around the origin. These are the relevant frequencies to describe long range properties of $\zeta_{t}$. To reproduce results parallel to Thm 2 of Robinson (1995a) or Thm 1 of Velasco (1999), for stationary and difference stationary processes respectively, we define a (possibly nonintegrable but) stationary analogue to a spectrum for $\zeta_{t}$,

$$
f_{\zeta \zeta}(\lambda) \quad f_{\eta \eta}(\lambda)|\phi(\lambda)|^{2}
$$

(see e.g. Velasco, 1999 for difference stationary processes). The spectrum $f_{\zeta \zeta}(\lambda)$ is nonintegrable for $\alpha \geq 0.5$ because we show in Lemma 1 (for $\alpha>0$ ) that

$$
f_{\zeta \zeta}(\lambda) \sim f_{\eta \eta}(0)|\lambda|^{-2 \alpha}, \quad \text { as } \lambda \rightarrow 0,
$$

with $0<f_{\eta \eta}(0)<\infty$. This spectrum describes the second order dynamic properties of $\zeta_{t}$ and would be the reference target for log periodogram regressions because it equals the limit of the expectation of the periodogram, as happens for (difference) stationary process (see also Solo, 1992). The spectrum $f_{\zeta \zeta}(\lambda)$ is the limit, as $n \rightarrow \infty$, of the time varying spectral density

$$
f_{\zeta \zeta}^{(n)}(\lambda) \quad f_{\eta \eta}(\lambda) \frac{1}{n} \sum_{t}^{n}\left|\phi_{t}(\lambda)\right|^{2}, \quad \phi_{t}(\lambda) \quad \sum_{j=0}^{t-1} \phi_{j}^{(\alpha)} \exp (\mathrm{i} j \lambda),
$$

which is directly related to the expectation of the periodogram $I_{\zeta \zeta}(\lambda) \quad\left|w_{\zeta}(\lambda)\right|^{2}$. To see this we can use that 


$$
E\left[w_{\zeta}\left(\lambda_{j}\right) w_{\zeta}\left(\lambda_{k}\right)\right] \quad \frac{1}{2 \pi n} \int_{\Pi} \chi_{j, k}(\mu) f_{\eta \eta}(\mu) \mathrm{d} \mu,
$$

see (8.1) in Robinson and Marinucci (2001), where $\Pi \quad[\pi, \pi]$ and

$$
\chi_{j, k}(\mu) \quad \phi_{n}\left(\lambda_{j}, \quad \mu\right) \phi_{n}\left(\lambda_{k}, \mu\right), \quad \phi_{n}(\lambda, \mu) \quad \sum_{t=1}^{n} \exp (\mathrm{it}(\lambda+\mu)) \phi_{t}(\mu) .
$$

Then we can check that

$$
\frac{1}{2 \pi n} \int_{\Pi} \chi_{j, j}(\mu) \mathrm{d} \mu \quad \frac{1}{n} \sum_{t=1}^{n}\left|\phi_{t}\left(\lambda_{j}\right)\right|^{2}
$$

and, if $f_{\eta \eta}(\mu)$ is smooth enough at $\mu \quad \lambda_{j}$, we can approximate the periodogram expectation $E\left[I_{\zeta \zeta}\left(\lambda_{j}\right)\right] \quad E\left[w_{\zeta}\left(\lambda_{j}\right) w_{\zeta}\left(\lambda_{j}\right)\right]$ by $f_{\zeta \zeta}^{(n)}\left(\lambda_{j}\right)$. Here, the function $\chi_{j, k}(\mu)$ plays the joint role of Fejer's kernel,

$$
K_{n}(\mu) \quad(2 \pi n)^{-1}\left|\sum_{t}^{n} \exp (\mathrm{i} t \mu)\right|^{2},
$$

and of the fractional transfer function

$$
|\varphi(\mu)|^{2} \quad\left|\sum_{j}^{\infty} \varphi_{j}^{(d)} \exp (\mathrm{ij} \mu)\right|^{2} \quad|1 \quad \exp (\mathrm{i} \mu)|^{-2 d}
$$

in the (difference) stationary cases (1) and (2) for $d<1.5$, since in this case

$$
E\left[I_{y y}\left(\lambda_{j}\right)\right] \quad \int_{\Pi} K_{n}\left(\lambda_{j} \quad \mu\right) f_{\varepsilon \varepsilon}(\mu)|\varphi(\mu)|^{2} \mathrm{~d} \mu .
$$

The kernel $K_{n}(\mu)$ tends to a Dirac's delta function $\delta(\mu)$ as $n \rightarrow \infty$, so the above quantity tends to $f_{y y}\left(\lambda_{j}\right) \quad f_{\varepsilon \varepsilon}\left(\lambda_{j}\right)\left|\varphi\left(\lambda_{j}\right)\right|^{2}$. The study of the properties of eqn (6) based on the kernel $\phi_{n}(\lambda, \mu)$ generalizes usual stationary Fourier analysis techniques for time dependent linear filters, possibly with algebraic decay to zero.

Now we impose some extra smoothness conditions on $f_{\zeta \zeta}(\lambda)$ at $\lambda \quad 0$ through $\phi$ $(\lambda)$ and $f_{\eta \eta}(\lambda)$.

\section{Assumption 2.}

1. $f_{\eta \eta}(\lambda)$ is boundedly differentiable for $\lambda \in(\epsilon, \epsilon)$, some $\epsilon>0$.

2. $\phi(\lambda)$ is differentiable in $(\epsilon, \epsilon) \quad\{0\}$, with

$$
\frac{\mathrm{d}}{\mathrm{d} \lambda} \phi(\lambda) \quad O\left(|\phi(\lambda) \| \lambda|^{-1}\right) \quad \text { as } \lambda \rightarrow 0 .
$$

This assumption is similar to Assumption 2 of Theorem 2 of Robinson (1995a), where the covariance matrix of the DFT of stationary long memory series is 
analysed. It does not impose extra smoothness compared with this case and it implies that $(\mathrm{d} / \mathrm{d} \lambda) \log f_{\zeta \zeta}(\lambda) \quad O\left(|\lambda|^{-1}\right)$ as $\lambda \rightarrow 0$, in view of Lemma $1(\alpha>0)$ and (2) of Assumption $1(\alpha<0)$. We give now an equivalent result for the DFT of $\zeta_{t}$ at Fourier frequencies, whose proof can be found in the Appendix. Here and elsewhere we use the notation

$$
(\log n)^{\alpha} 0.5 \quad\left\{\begin{array}{lll}
\log n & \text { if } \alpha & 0.5 \\
1 & \text { otherwise. }
\end{array}\right.
$$

THeORem 1. Under Assumptions 1 and 2, for $1 \leq k<j<m, m / n \rightarrow 0$ as $n \rightarrow \infty$,

1. If $0<\alpha<1$, then we have

$$
\begin{aligned}
& E\left[w_{\zeta}\left(\lambda_{j}\right) w_{\zeta}\left(\lambda_{j}\right)\right] \quad f_{\zeta \zeta}\left(\lambda_{j}\right)+O\left(f_{\zeta \zeta}\left(\lambda_{j}\right)\left\{j^{\alpha-1}+j^{-1}(\log n)^{\alpha}{ }^{0.5}\right\}\right) ; \\
& E\left[w_{\zeta}\left(\lambda_{j}\right) w_{\zeta}\left(\lambda_{k}\right)\right], \quad E\left[w_{\zeta}\left(\lambda_{j}\right) w_{\zeta}\left(\lambda_{k}\right)\right], \quad E\left[w_{\zeta}\left(\lambda_{j}\right) w_{\zeta}\left(\lambda_{j}\right)\right] \\
& O\left(\sqrt{f_{\zeta \zeta}}\left(\lambda_{j}\right) f_{\zeta \zeta}\left(\lambda_{k}\right)\left\{j^{\alpha-1}+\left(\frac{j}{k}\right)^{\alpha} n^{-1} \log j+j^{-1}(\log n)^{\alpha} \quad 0.5\right\}\right) .
\end{aligned}
$$

2. If $1<\alpha<0$, then we have

$$
\begin{aligned}
E\left[w_{\zeta}\left(\lambda_{j}\right) w_{\zeta}\left(\lambda_{j}\right)\right] & f_{\zeta \zeta}\left(\lambda_{j}\right) \\
& +O\left(f_{\zeta \zeta}\left(\lambda_{j}\right)\left\{j^{-1}+n^{-1} \log j+j^{-2|\alpha|} n^{2|\alpha|-1}(\log n)^{\alpha}-0.5\right\}\right) ; \\
E\left[w_{\zeta}\left(\lambda_{j}\right) w_{\zeta}\left(\lambda_{k}\right)\right], & E\left[w_{\zeta}\left(\lambda_{j}\right) w_{\zeta}\left(\lambda_{k}\right)\right], \quad E\left[w_{\zeta}\left(\lambda_{j}\right) w_{\zeta}\left(\lambda_{j}\right)\right] \\
O\left(\sqrt { f _ { \zeta \zeta } } ( \lambda _ { j } ) f _ { \zeta \zeta } ( \lambda _ { k } ) \left\{j^{-1}+\left(\frac{j}{k}\right)^{|\alpha|} n^{-1} \log j+j^{-2|\alpha|} n^{2|\alpha|-1}(\log n)^{\alpha}\right.\right. & -0.5\}) .
\end{aligned}
$$

3. For $\propto 0$ all the bounds are replaced by $O\left(\sqrt{ } f_{\zeta \zeta}\left(\lambda_{j}\right) f_{\zeta \zeta}\left(\lambda_{k}\right) k^{-1} \log j\right), \quad k \leq j$.

The bounds obtained in this theorem for the normalized periodogram expectation $f_{\zeta \zeta}^{-1}\left(\lambda_{j}\right) E\left[I_{\zeta \zeta}\left(\lambda_{j}\right)\right] \quad 1$ of nonstationary data at Fourier frequencies $\lambda_{j}$ are different for any $\alpha / 0$ from the stationary ones, $O\left(j^{-1} \log j\right), \quad 0.5<\alpha<0.5$, given by Robinson (1995a) and reported here for the $\alpha 0$ case. They are also different from the difference stationary result, $O\left(j^{2(\alpha-1)} \log j\right.$ ), $0.5 \leq \alpha<1$ (see Velasco, 1999), but dependent on the degree of integration as well. For instance, the new contribution in $j^{\alpha-1}$ when $\alpha>0$ arises from the temporal inhomogeneity of $\zeta_{t}, t$ $1, \ldots, n$, when the time invariant spectral density $f_{\zeta \zeta}(\lambda)$ is compared with the time varying spectral density $f_{\zeta \zeta}^{(n)}(\lambda)$. However, when $0.5<\alpha<0$ the results in Theorem 1 are the same as in the stationary case, up to a logarithmic factor, and as expected, the effects of truncation seem to be alleviated in case of negative memory.

Robinson (2005) obtained for fractional series $\zeta_{t}$ as in eqn (3) that

$$
f_{\zeta \zeta}^{-1}\left(\lambda_{j}\right) E\left[\left|w_{\zeta}\left(\lambda_{j}\right) \quad w_{x}\left(\lambda_{j}\right)\right|^{2}\right] \quad O\left(j^{-1}(\log n)^{\alpha-0.5}\right)
$$


for $\alpha \in[0.5,0.5)$ when $x_{t}$ is given by eqn (1) for $d \equiv \alpha$ and the same sequence of innovations. Using this result and a triangle inequality in his equation (C.4), together with Robinson (1995a, Thm 2(i)), we can obtain that for $\alpha \in(0,0.5)$, $f_{\zeta \zeta}^{-1}\left(\lambda_{j}\right) E\left[I_{\zeta \zeta}\left(\lambda_{j}\right)\right] \quad 1+O\left(j^{-1 / 2}\right)$. This result is improved by the corresponding bound $O\left(j^{\alpha-1}\right)$ in our Theorem 1 for any $\alpha<0.5$. This improvement is important to allow for the asymptotic normality of the log periodogram regression estimate of $\alpha$, for $|\alpha|<0.5$, but only consistency can be obtained when $\alpha \geq 0.5$ (see Section 3). Using the same arguments in the proof of Theorem 1 and considering only the contribution from $\left|\phi_{t}(\lambda)\right|^{2}$ in eqn (21), we can obtain under Assumptions 1 and 2 that the left hand side of eqn (7) is $O\left(j^{-1}+n^{-1} \log j\right)$ for $\alpha \in(0,0.5)$, see eqn (24), which generalizes Robinson's (2005) result to long memory filters in the class $\Phi(\alpha)$ by means of local assumptions on the smoothness of $f_{\eta \eta}(\lambda)$ and $\phi(\lambda)$ at $\lambda \quad 0$.

Phillips (1999) provides some probability bounds on the difference $w_{\zeta}\left(\lambda_{j}\right) \quad\left(1 \quad \mathrm{e}^{i \lambda_{j}}\right)^{-\alpha} w_{\eta}\left(\lambda_{j}\right), \quad \alpha>0$, for fractionally integrated $\zeta_{t}$ at each frequency $\lambda_{j}$, either fixed or in a degenerating band. Apart from some correction terms, similar to the ones discussed in Section 4 below, which are $O_{p}\left(\lambda_{j}^{-1} n^{-1 / 2}\right)$ when $\alpha<0.5$, the remainder is $O_{p}\left(\lambda_{j}^{-\alpha} j^{\alpha-1}\right)$, which would correspond to a similar bound to the one in (1) of our Theorem $1, \alpha>0$. Taking those bounds as uniform, some properties of different estimates are deduced in Phillips (1999), and some ad hoc modifications of the usual semiparametric procedures of memory estimation can be proposed (see also Kim and Phillips, 1999). However, note that for the analysis of the properties of statistics which are nonlinear functions of the periodogram of an observed fractionally integrated series, such as the log periodogram memory estimate studied in Section 3, the analysis of different approximations of the DFT of data are not sufficient. Uniform bounds on the periodogram expectations of $\zeta_{t}$ under Gaussianity are required in this case because it is not enough to control the (linear) distance with the DFT of the stationary version of the original process [cf. eqn (7)].

For the analysis of the periodogram properties at all frequencies in $[0, \pi]$, including those fixed for which $j$ is of the same order as $n$, we need to make Assumption 2 uniform for any $\lambda \in[0, \pi]$. Then the results of Theorem 1 will hold and full parametric estimates for nonstationary trending data could be studied as in Velasco and Robinson (2000). In Section 3, we concentrate on a particular semi parametric estimate under our local conditions.

\section{THE LOG-PERIODOGRAM REGRESSION ESTIMATE OF $\alpha$}

The semiparametric estimate of the memory parameter $\alpha$ based on the log periodogram regression estimate was proposed by Geweke and Porter Hudak (1983). Robinson (1995a) showed that the periodogram of stationary long 
memory series is asymptotically unbiased and uncorrelated, as with short memory, when evaluated at the harmonic frequencies $\lambda_{j}$ for $j$ growing with sample size $n$. This is the basis to write the logarithm of eqn (5) as a linear regression model with approximately homoscedastic and uncorrelated errors,

$$
\log I_{\zeta \zeta}\left(\lambda_{j}\right) \approx \log f_{\eta \eta}(0) \quad 2 \alpha \log \lambda_{j}+\log \frac{I_{\zeta \zeta}\left(\lambda_{j}\right)}{f_{\zeta \zeta}\left(\lambda_{j}\right)}, \quad j \quad 1, \ldots, m,
$$

where $m$ is small compared with $n$. The log periodogram regression estimate $\hat{\alpha}$ is the least squares estimate of $\alpha$,

$$
\left.\hat{\alpha} \quad\left(\begin{array}{ll}
\sum_{j}^{m}\left(r_{j}\right. & r
\end{array}\right)^{2}\right)^{-1} \sum_{j=1}^{m}\left(r_{j} r\right) \log I_{\zeta \zeta}\left(\lambda_{j}\right),
$$

where the log periodogram is the dependent variable and the regressor is $r_{j}$ $2 \log \lambda_{j}, r m^{-1} \sum_{j 1}^{m} r_{j}$. The asymptotic properties of $\hat{\alpha}$ were analysed rigorously for multiple stationary Gaussian series $(0.5<\alpha<0.5)$ by Robinson (1995a). He considered a pooling of contributions from adjacent frequencies to achieve efficiency gains and showed that the periodograms at the very first frequencies do not have nice asymptotic statistical properties, so following Künsch (1987) he proposed to exclude the first $\ell$ frequencies from the regression. Hurvich et al. (1998), HDB henceforth, have shown under some additional conditions that trimming of very low frequencies may not be necessary for the analysis of the asymptotic properties of $\hat{\alpha}$. HDB only consider fractional processes with weakly dependent innovations which possess a spectral density with three bounded derivatives at $\lambda \quad 0$, and the regressor in eqn (8) which arises naturally for fractional processes, $z_{j} \log \left(4 \sin ^{2}\left(\lambda_{j} / 2\right)\right)$, as originally proposed by Geweke and Porter Hudak. However, we generalized their results under the following assumption. We say that a function $g(x)$ is $\operatorname{Hölder}(\tau), 0<\tau \leq 1$, in an interval of the origin if $|g(x) \quad g(0)| \leq C|x|^{\tau}$ uniformly for $x \in(\epsilon, \epsilon)$, for some $\epsilon>0$ and $C<\infty$.

Assumption 3. In addition to Assumption 2, we assume

1. If $1<\rho \leq 2$, then $f_{\eta \eta}(\lambda)$ is differentiable and $f_{\eta \eta}^{\prime}(\lambda)$ is Hölder $(\rho \quad 1)$ in an open interval of the origin. If $2<\rho \leq 3$ then $f_{\eta \eta}(\lambda)$ is twice differentiable and $f_{\eta \eta}^{\prime \prime}(\lambda)$ is Hölder $(\rho \quad 2)$ in an open interval of the origin.

2. If $0<\gamma \leq 2$, then $|\phi(\lambda)|^{2} \quad|\lambda|^{-2 \alpha}\left(1+O\left(|\lambda|^{\gamma}\right)\right)$ as $\lambda \rightarrow 0$. If $2<\gamma \leq 3$, then $|\phi(\lambda)|^{2} \quad|\lambda|^{-2 \alpha}\left(1+A_{\alpha} \lambda^{2}+O\left(|\lambda|^{\gamma}\right)\right)$ as $\lambda \rightarrow 0$.

Assumption 3 imposes a rate on the approximation (5), being 3. of Assumption 3 similar to Robinson's (1995a) Assumption 1. The reason for considering values of $\rho$ and $\gamma$ larger than 2 is to estimate explicitly the bias of $\hat{\alpha}$, although consistency requires only $\min \{\rho, \gamma\}>0$ and the best possible rate for the asymptotic bias is already achieved by $\min \{\rho, \gamma\} \quad 2$. Thus for fractional time series, we have that 


$$
|\phi(\lambda)|^{2} \quad\left(2 \sin \frac{\lambda}{2}\right)^{-2 \alpha}|\lambda|^{-2 \alpha}\left(1+\frac{\alpha}{12} \lambda^{2}+O\left(|\lambda|^{3}\right)\right), \quad \text { as } \lambda \rightarrow 0,
$$

so $\gamma \quad 3$ and following Robinson (1995a), for these models we have that

$$
f_{\zeta \zeta}(\lambda) \quad f_{\eta \eta}(0)|\lambda|^{-2 \alpha}\left(1+\left[\frac{f_{\eta \eta}^{\prime \prime}(0)}{f_{\eta \eta}(0)}+\frac{\alpha}{12}\right] \lambda^{2}+O\left(|\lambda|^{\rho}\right)\right), \quad \text { as } \lambda \rightarrow 0,
$$

for $\rho>2$, using that $f_{\eta \eta}^{\prime}(0) \quad 0$. For example, the presence of additive noise in $\zeta_{t}$ of smaller order of integration than $\alpha$ would lead to values of $\gamma$ smaller than 2. Therefore, we can adapt the bias estimation results of HDB, although they defined the log periodogram regression estimate using the fractional regressors $z_{j}$.

Theorem 2. Let $0.5<\alpha<0.5$. Under Assumptions $13, \delta \min \{\rho, \gamma\} \in$ $(2,3]$, and

$$
m^{-1}+\left(m n^{-1}\right)^{\delta-2} \log m \rightarrow 0 \quad \text { as } n \rightarrow \infty
$$

for Gaussian $\eta_{t}$

$$
\begin{aligned}
& E(\hat{\alpha}) \quad \alpha \quad \frac{2 \pi^{2}}{9}\left[\frac{f_{\eta \eta}^{\prime \prime}(0)}{f_{\eta \eta}(0)}+A_{\alpha}\right] \frac{m^{2}}{n^{2}}(1+o(1))+\delta_{m} \\
& \operatorname{var}(\hat{\alpha}) \quad \frac{\pi^{2}}{24 m}+o\left(m^{-1}\right),
\end{aligned}
$$

where $\delta_{m}: \quad O\left(m^{\alpha-1} \log m\right) 1_{\alpha>0}+O\left(m^{-1} \log ^{2} m\right) 1_{\alpha<0}+O\left(m^{-1} \log ^{3} m\right) 1_{\alpha} 0$.

Proof of Theorem 2. Follows from HDB, just using our Theorem 1 instead of Theorem 2 or Robinson (1995a). Then all Lemmas of HDB hold under the conditions of the theorem, just setting in their Lemma 3 that $\alpha_{j k} j^{\alpha-1}+$ $(j / k)^{\alpha} n^{-1} \log j, \quad 0<\alpha<0.5, \log m \leq k<j \leq m, m^{-1}+m n^{-1} \rightarrow 0$ and the corresponding modifications for $\alpha \leq 0$ from Theorem 1. Then HDB's Lemma 4 follows now from the appropriate modification of Lem 4.1 of Robinson and Marinucci (2001). In Lemmas 6 and 7 of HDB we obtain that $E\left[\epsilon_{j}\right]$ $\alpha_{j j}$ where $\alpha_{j j} \quad O\left(j^{\alpha-1}\right), \alpha>0 ; \alpha_{j j} \quad O\left(j^{-1}\right), \alpha<0$, and $\operatorname{var}\left[\epsilon_{j}\right] \quad \pi^{2} / 6+O\left(\alpha_{j j}\right)$, $\log m \leq j \leq m$, whereas in Lem 8 of HDB, the bound is now $O\left(m^{\alpha-1} \log m\right)$, $\alpha>0 ; O\left(m^{-1} \log ^{2} m\right), \alpha<0$, under eqn (9). The condition $\left(m n^{-1}\right)^{\delta-2} \log m \rightarrow 0$ and our Hölder assumption are used instead of three bounded derivatives in HDB, while the condition $m n^{-1} \log m \rightarrow 0$ in HDB is implied by eqn (9), $\delta \leq 3$.

QED

If we use $z_{j}$ instead of $r_{j}$ in the log periodogram regression and $|\phi(\lambda)|^{2}$ $\left|1 \quad \mathrm{e}^{\mathrm{i} \lambda}\right|^{2}$, then the term $A_{\alpha}$ does not show up in Theorem 2 . When $\alpha \geq 0.5$, it is not possible to show the $\sqrt{ } m$ consistency of $\hat{\alpha}$, but just adapting the methods of the previous theorem we can prove the following result which gives conditions on $m$ for the consistency of $\hat{\alpha}$ for any value $\delta>0$ and $0.5<\alpha<1, \alpha / 0.5$. 
Theorem 3. Let $0.5<\alpha<1, \alpha / 0.5$. Under Assumptions $13, \delta$ $\min \{\rho, \gamma\} \in(0,2]$, and

$$
m^{-1}+\left(m n^{-1}\right)^{\delta} \log m \rightarrow 0 \quad \text { as } n \rightarrow \infty,
$$

for Gaussian $\eta_{t}$

$$
\begin{aligned}
E(\hat{\alpha}) \quad \alpha & O\left(\left(m n^{-1}\right)^{\delta} \log m\right)+\delta_{m}, \\
\operatorname{var}(\hat{\alpha}) & O\left(m^{-1}\right)+O\left(m^{2(\alpha-1)} \log ^{2} m\right) .
\end{aligned}
$$

The bandwidth conditions (9) and (10) hold if $m \sim K n^{a}$ for $0<a<1$. However, we are not able to show the consistency when $\alpha \quad 0.5$ and $\alpha \leq 0.5$ and all Fourier frequencies $\lambda_{1}, \ldots, \lambda_{m}$ are used because of the terms growing with $\log n$ and $n$ in Theorem 1 . However, if we wish to trim out the first $\ell$ frequencies in the log periodogram regression, for some $\ell$ growing slowly with $m$ but faster than $\log n$ in the asymptotics, then it is easy to adapt HDB's arguments and a similar result to Theorem 3 is valid also for $\alpha \quad 0.5$ and $\alpha \leq 0.5$. Finally, if $0.5<\alpha<0.5$ we can adapt Robinson's (1995a) central limit theorem as in HBD to avoid any trimming of low frequencies, and using a bandwidth $m \sim K n^{a}$ such that $0<a<2 \delta /(2 \delta+1)$.

Theorem 4. Let $0.5<\alpha<0.5$. Under Assumptions $13, \delta \min \{\rho, \gamma\} \in$ $(0,2]$, and

$$
m^{-1} \log ^{2} n+m^{2 \delta+1} n^{-2 \delta} \rightarrow 0 \quad \text { as } n \rightarrow \infty
$$

for Gaussian $\eta_{t}$

$$
\sqrt{ } m(\hat{\alpha} \quad \alpha) \rightarrow_{d} N\left(0, \frac{\pi^{2}}{24}\right)
$$

Proof of Theorem 4. Follows as Thm 2 of HDB from Robinson (1995a), adapting for a general smoothness condition on $f_{\eta \eta}(\lambda)$ in terms of $\delta$.

QED

The analysis of non Gaussian and tapered series can also be pursued using the ideas put forward here (see Velasco, 2000; Hurvich et al., 2002).

\section{PERIODOGRAM MODIFICATIONS AND INITIAL CONDITIONS}

We discuss some modifications of the periodogram proposed in the literature, which are alternatives to the usual detrending procedure of nonstationary series consisting on taking differences. We also briefly explore the implications of the definition of fractionally integrated processes in terms of a truncated filter in the previous asymptotic theory. 
Phillips (1999) proposed the following correction for the DFT at each $\lambda_{j}$ using a representation of the DFT that involves the last observation $\zeta_{n}$ (we assume no initial conditions, $X_{0} \equiv 0$ in his notation),

$$
w_{\zeta^{*}}\left(\lambda_{j}\right): \quad w_{\zeta}\left(\lambda_{j}\right)+\frac{1}{\sqrt{ } 2 \pi n} \frac{\mathrm{e}^{i \lambda_{j}}}{1} \mathrm{e}^{i \lambda_{j}} \zeta_{n}, \quad j / 0 \bmod n .
$$

Phillips(1999) and Kim and Phillips (1999) motivated such correction in terms of improving the properties of the DFT for trending processes with memory around $\alpha 1$, and it is suitable to deal with the cases where $\alpha \geq 1$, since in this case the usual log periodogram regression estimate is not consistent. In fact, this correction can be seen as a linear detrending in the time domain setting

$$
\zeta_{t}^{*} \quad \zeta_{t} \quad \frac{t}{n} \zeta_{n}, \quad t \quad 1, \ldots, n,
$$

so $\zeta_{n}^{*} \quad 0$ and $w_{\zeta}\left(\lambda_{j}\right)$ is actually the DFT of $\zeta_{t}^{*}$. If we set additionally $\zeta_{1} \quad 0$, so $\left(\begin{array}{lll}1 & L\end{array}\right) \zeta_{1} \quad 0$, it is easy to check that

$$
w_{\zeta^{*}}\left(\lambda_{j}\right) \quad w_{\Delta \zeta}\left(\lambda_{j}\right) \frac{\mathrm{e}^{\mathrm{i} \lambda_{j}}}{1 \quad \mathrm{e}^{\mathrm{i} \lambda_{j}}},
$$

for the increments $\Delta \zeta_{t} \quad\left(\begin{array}{ll}1 & L\end{array}\right) \zeta_{t}$. Therefore, the analysis of Sections 2 and 3 is valid for nonstationary series with $1 \leq \alpha<2$ replacing $\alpha$ by $\alpha^{*} \quad 1 \quad \alpha$ because, according to eqn (5), $\Delta \zeta_{t}$ has memory $\alpha^{*}$ and we can set $\phi_{\zeta}(\lambda)$ : $\mathrm{e}^{\mathrm{i} \lambda}\left(1 \quad \mathrm{e}^{i \lambda}\right)^{-1} \phi_{\Delta \zeta}(\lambda)$ where $\phi_{\Delta \zeta}(\lambda) \quad\left(1 \quad \mathrm{e}^{\mathrm{i} \lambda}\right) \phi_{\zeta}(\lambda)$. In this sense the transfor mation (10) is equivalent to taking first differences, and higher order corrections can be developed for further differencing. However, when $0<\alpha<1$ and the modified DFT (11) is used instead of $w_{\zeta}\left(\lambda_{j}\right)$, the implicit process $\zeta_{t}^{*}$ has negative memory (because $\Delta \zeta_{t}$ typically has) and the properties of its DFT and log periodogram estimates are immediate consequences of our previous results with $\alpha<0$.

We now explore the initial conditions problem. Let us assume for some $T \geq 1$ the following model for $\zeta_{t} \quad \zeta_{t}(T)$ :

$$
\zeta_{t} \quad \sum_{j-T}^{t} \phi_{t-j}^{(\alpha)} \eta_{j}
$$

This includes the set ups of Robinson and Marinucci (2001), $T$ 1; Phillips (1999), $T \quad 0$, and other possibilities with $T$ fixed with $n$ or with $T$ increasing with $n$, e.g. $T \quad n^{b}, b>0$, which implies that the stretch of initial conditions increases with the window of observed data. As far as $T$ is fixed, it is easy to check in the Appendix that all the results of Sections 2 and 3 hold as if $T \quad 1$ just modifying the bound for $|\phi(\lambda)|^{2} \quad\left|\phi_{t+T+1}(\lambda)\right|^{2}$ used in the proof of Theorem 1. We only consider the case where $\alpha \geq 0$, since negative values of $\alpha$ usually originate from some differencing process given a sample of size $n$. 
COROLlary 1. The conclusions of Theorems 14 are valid for any $T \geq 0, \alpha \geq 0$.

In fact, if $T$ is fixed or $O(n)$, the asymptotic bounds given in Theorem 1 cannot be easily improved further, but if $T$ is increasing fast enough with $n$, some improvements seem possible. In particular, the bound $O\left(j^{\alpha-1}\right)$ in Theorem 1 , $\alpha>0$, can be multiplied by a factor $\left(n T^{-1}\right)^{1-\alpha}$ which is $o(1)$ as $n \rightarrow \infty$ if $n$ $o(T), 0<\alpha<1$.

Note that if $T n^{-1} \rightarrow \infty$ as $n \rightarrow \infty$ (initial conditions growing faster than observed data) then we are closer to the (difference) stationary framework of Hurvich and Ray (1995) and Velasco (1999), and the contribution of the past innovations to each observation $\zeta_{t}$,

$$
P_{t}(T): \quad \sum_{j}^{0} \phi_{t-j}^{(\alpha)} \eta_{j} \quad \sum_{j}^{T+t} \phi_{j}^{(\alpha)} \eta_{t-j},
$$

can be showed to be $O_{p}\left(t^{2 \alpha-1}\right), 0<\alpha<0.5$; or $O_{p}\left((T+t)^{2 \alpha-1}\right)$ for $\alpha \geq 0.5$, under some regularity conditions on $\eta_{t}$. Thus, assuming that $\operatorname{cov}\left[\eta_{j}, \eta_{k}\right]$ $O\left(\begin{array}{ll}j & \left.k\right|^{-\delta}\end{array}\right), \delta>1$, we have that

$$
\begin{aligned}
\operatorname{var}\left[P_{t}(T)\right] & \sum_{j}^{T+t} \sum_{k}^{T+t} \phi_{j}^{(\alpha)} \phi_{k}^{(\alpha)} \operatorname{cov}\left[\eta_{t-j}, \eta_{t-k}\right] \\
\leq & C \sum_{j t}^{T+t} \sum_{k}^{T+t} j^{\alpha+1} j^{\alpha-1} k^{\alpha-1}|j \quad k|^{-\delta} \\
\leq & C \sum_{j t}^{T+t} j^{2(\alpha-1)} \sum_{k}^{T+t-j} k^{-\delta} \leq C^{*} \sum_{j t}^{T+t} j^{2(\alpha-1)},
\end{aligned}
$$

and the claim follows. For trending observations with $\alpha>0.5$, the time dependent initial conditions can have a dominant contribution compared with the information accumulated in the current periods $1,2, \ldots, t$, as long as $t / T \rightarrow 0$.

\section{FRACTIONAL INTEGRATION AND DIFFERENCING OF STATIONARY} LONG-RANGE-DEPENDENT TIME SERIES

We consider the situation where a finite observed stretch, $t \quad 1, \ldots, n$, of a stationary long memory time series $x_{t}$, with memory $0.5<d<0.5$ is fractionally integrated (differenced) of order $\alpha>0(\alpha<0)$. These are the basic operations performed when using fractional values of $\alpha$ on both asymptotically stationary and trending processes, where we use an estimate $\alpha$ close to $d$ such that the filtered series is near stationary and short range dependent. We only consider $0.5 \leq \alpha<0.5$ since the fractional filters are cumulative in the sense that if $\phi_{t}^{(\alpha)} \in \Phi(\alpha)$, and $\phi_{t}^{(\beta)} \in \Phi(\beta), \alpha, \beta>0$ then 


$$
\sum_{j}^{t} \phi_{j}^{(\alpha)} \phi_{t-j}^{(\beta)} \in \Phi(\alpha+\beta)
$$

(cf. Robinson and Marinucci's, 2001 Lem 3.1), as motivated in Section 2 when presenting the filters with negative $\alpha$. Otherwise, we can first integrate or take differences, and the properties of the DFT of these processes can be obtained as a direct consequence of the results of this section.

Assumption 4. For $\left\{\phi_{t}^{(\alpha)}\right\} \in \Phi(\alpha)$ as in Assumption $1,|\alpha|<1$,

with

$$
\zeta_{t} \quad \sum_{j=1}^{t} \phi_{t-j} x_{j},
$$

and where $x_{t}$ is covariance stationary with spectral density satisfying $f_{x x}(\lambda)$ $O\left(|\lambda|^{-2 d}\right), \quad 0.5 \leq d<0.5$, and

$$
\frac{\mathrm{d}}{\mathrm{d} \lambda} f_{x x}(\lambda) \quad O\left(f_{x x}(\lambda)|\lambda|^{-1}\right) \quad \text { as } \lambda \rightarrow 0 .
$$

The class of processes $x_{t}$ defined Assumption 4 is more general than eqn (1), since we do not require $x_{t}$ to be a fractional series, only to have a spectral density with a similar asymptotic behaviour as $\lambda \rightarrow 0$. Therefore, the set of fractionally integrated processes $\zeta_{t}$ described by Assumption 4 is more general than that of Phillips (1999) where only uniformly smooth spectral densities $f_{x x}$ are considered, so $x_{t}$ is necessarily short memory. Using our previous definition, it holds that

$$
f_{\zeta \zeta}(\lambda) \quad|\phi(\lambda)|^{2} f_{x x}(\lambda) \sim G|\lambda|^{-2(d+\alpha)} \quad \text { as } \lambda \rightarrow 0,
$$

and to estimate the exponent $h: \quad d+\alpha$ we analyse the asymptotic properties of the moments of the DFT of long memory $\alpha$ fractionally integrated or differenced time series. To detrend an observed series, then ideally $\alpha \approx d$, so $h \approx 0$, and upper bounds on $h$ should not be very restrictive in practical applications. The following theorems can be considered typically for series with $d<0(\alpha>0)$ and $d>0$ $(\alpha<0)$ respectively, for which $h$ is small. The proof is in the Appendix.

Theorem 5. Under Assumption 4, $d \in[$ 0.5, 0.5), $|h|<0.5, h d+\alpha$, for $1 \leq k<j<m, m / n \rightarrow 0$ as $n \rightarrow \infty$,

1. When $0<\alpha<0.5$, then we have

$$
\begin{aligned}
& E\left[w_{\zeta}\left(\lambda_{j}\right) w_{\zeta}\left(\lambda_{j}\right)\right] \quad f_{\zeta \zeta}\left(\lambda_{j}\right)+O\left(f_{\zeta \zeta}\left(\lambda_{j}\right) j^{\alpha-1}\right) ; \\
& E\left[w_{\zeta}\left(\lambda_{j}\right) w_{\zeta}\left(\lambda_{k}\right)\right], \quad E\left[w_{\zeta}\left(\lambda_{j}\right) w_{\zeta}\left(\lambda_{k}\right)\right], \quad E\left[w_{\zeta}\left(\lambda_{j}\right) w_{\zeta}\left(\lambda_{j}\right)\right] \\
& O\left(\sqrt{f_{\zeta \zeta}}\left(\lambda_{j}\right) f_{\zeta \zeta}\left(\lambda_{k}\right)\left[j^{-1}(\log n)^{\alpha-0.5}+\left(\frac{j}{k}\right)^{|\alpha|+|d|} j^{-1} \log j\right]\right) \text {. }
\end{aligned}
$$


2. When $0.5 \leq \alpha<0$, then we have

$$
\begin{aligned}
& E\left[w_{\zeta}\left(\lambda_{j}\right) w_{\zeta}\left(\lambda_{j}\right)\right] \quad f_{\zeta \zeta}\left(\lambda_{j}\right)+O\left(f_{\zeta \zeta}\left(\lambda_{j}\right) j^{-1}(\log n)^{\alpha-0.5}\right) ; \\
& E\left[w_{\zeta}\left(\lambda_{j}\right) w_{\zeta}\left(\lambda_{k}\right)\right], \quad E\left[w_{\zeta}\left(\lambda_{j}\right) w_{\zeta}\left(\lambda_{k}\right)\right], \quad E\left[w_{\zeta}\left(\lambda_{j}\right) w_{\zeta}\left(\lambda_{j}\right)\right] \\
& O\left(\sqrt{ } f_{\zeta \zeta}\left(\lambda_{j}\right) f_{\zeta \zeta}\left(\lambda_{k}\right)\left[j^{-1}(\log n)^{\alpha-0.5}+\left(\frac{j}{k}\right)^{|\alpha|+|d|} j^{-1} \log j\right]\right) .
\end{aligned}
$$

3. For $\propto \quad 0$, all the bounds are $O\left(\sqrt{ } f_{\zeta \zeta}\left(\lambda_{j}\right) f_{\zeta \zeta}\left(\lambda_{k}\right) k^{-1} \log j\right), k \leq j$.

Note that when $d \quad 0$ we basically recover the bounds of Theorem 1 , just changing $n$ by $j$ in the second part of this theorem, because Assumption 4 replaces the bounded differentiability of $f_{\eta \eta}$ at the origin, so now $(\mathrm{d} / \mathrm{d} \lambda) \log f_{x x}(\lambda)$ $O\left(|\lambda|^{-1}\right)$. Furthermore, we also recover the results from Robinson's (1995a) Thm 2 for stationary long memory time series $\left(\begin{array}{ll}\alpha & 0\end{array}\right)$, except for the term $j^{\alpha-1}$ when $\alpha>0$, so it is still possible to reproduce all the results of HBD on the asymptotic properties of the log periodogram estimate of the memory $h \alpha+d$ for stationary Gaussian time series with memory $d$ possibly different from zero,

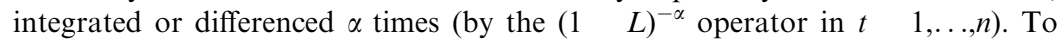
estimate the mean squared error and obtain the asymptotic distribution of $\hat{h}$ we introduce this new assumption, which generalizes Assumption 3. We omit the proof because it is similar to that of previous results.

Assumption 5. In addition to Assumption 4, we set $f_{x x}(\lambda) \quad|\lambda|^{-2 \mathrm{~d}} \mathrm{f}_{\mathrm{xx}}^{*}(\lambda)$ and assume

1. If $1<\rho \leq 2$, then $f_{x x}^{*}(\lambda)$ is differentiable and $f_{x x}^{* \prime}(\lambda)$ is $\operatorname{Hölder}(\rho \quad 1)$, $\lambda \in(0, \epsilon), \epsilon>0$. If $2<\rho \leq 3$, then $f_{x x}^{*}(\lambda)$ is twice differentiable and $f_{x x}^{* \prime \prime}(\lambda)$ is $\operatorname{Hölder}(\rho \quad 2), \lambda \in(0, \epsilon), \epsilon>0$.

2. If $0<\gamma \leq 2$, then $|\phi(\lambda)|^{2} \quad|\lambda|^{-2 \alpha}\left(1+O\left(|\lambda|^{\gamma}\right)\right)$ as $\lambda \rightarrow 0$. If $2<\gamma \leq 3$, then $|\phi(\lambda)|^{2} \quad|\lambda|^{-2 \alpha}\left(1+A_{\alpha} \lambda^{2}+O\left(|\lambda|^{\gamma}\right)\right)$ as $\lambda \rightarrow 0$.

Theorem 6. Let $|\alpha|<0.5, h \quad d+\alpha \in(0.5,0.5)$. Then the conclusions of Theorems 2 and 4 hold for the log periodogram estimate $\hat{h}$ of Gaussian series $\zeta_{t}(12)$ under Assumption 5 (replacing $f_{\eta \eta}$ by $f_{x x}^{*}$ ), for $\tau \in(2,3]$ and $\tau \in(0,2]$ respectively, $\tau \min \{\rho, \gamma\}$, and the same choices of $m$.

The theory developed does not include the situations where an initial estimate $\hat{d}_{n}$ is computed and the sequence $\Delta^{-\hat{d}_{n}} \zeta_{t}, t \quad 1, \ldots, n$, is obtained by fractional differencing. This operation should approach the weak dependent innovations $\Delta^{-d \zeta_{t}}$ if $\hat{d}$ converges fast enough to the true $d$, but in general the limit might depend on the distribution of $\hat{d}$. This problem also relates to memory estimation based on residuals, obtained either from deterministic time detrending or by stochastic detrending in cointegrated systems, as is analysed in Marmol and 
Velasco (2001) and Hassler et al. (2006) respectively. Similar techniques to those of this paper can also be used for the analysis of long range dependent time series generated by different simulation algorithms.

\section{APPENDIX}

PROOFS OF RESULTS

In Lemma 1 we collect some technical results about $\phi(\lambda)$ and $\phi_{t}(\lambda)$ to be used in the proof of the results contained in this appendix and which can be of independent interest for Fourier analysis with nonstationary filters. Note that the bound for $\phi_{n}$ improves upon (8.3) of Robinson and Marinucci (2001), what is key for many of our results. Lemma 2 below will extend these results for $\alpha<0$. In the sequel $C$ denotes a generic constant that may change each time is used.

Lemma 1. Under Assumption 1, for $\left\{\phi_{t}^{(\alpha)}\right\} \in \Phi(\alpha), \quad 0<\alpha<1$, as $|\lambda| \rightarrow 0$, $|\phi(\lambda)|^{2} \sim|\lambda|^{2 \alpha} ; \quad\left|\phi_{t}(\lambda)\right| \leq C \min \left\{t^{\alpha},|\lambda|^{\alpha}\right\} ; \quad\left|\phi_{t}(\lambda)\right| \leq C \min \left\{t^{\alpha}{ }^{1}|\lambda|^{1},|\lambda|{ }^{\alpha}\right\}, \quad$ where $\bar{\phi}_{t}(\lambda) \quad \phi(\lambda) \quad \phi_{t}(\lambda) ;$ and $\left|\phi_{n}(\lambda, \mu)\right| \leq C \min \left\{n,|\lambda+\mu|^{1}\right\} \min \left\{n^{\alpha},\left[|\mu|^{\alpha}+|\lambda|^{\alpha}\right]\right\}$.

Proof of Lemma 1. The bounds for $|\phi(\lambda)|^{2},\left|\phi_{t}(\lambda)\right|$ and $\left|\bar{\phi}_{t}(\lambda)\right|$ are a direct consequence of Lemma 3.2 in Robinson and Marinucci (2001). To obtain the bounds for $\phi_{n}(\lambda, \mu)$, first we have that, with $\left|\phi_{t}(\lambda)\right| \leq C t^{\alpha}$,

$$
\left|\phi_{n}(\lambda, \mu)\right| \quad\left|\sum_{t}^{n} \mathrm{e}^{\mathrm{i} t(\lambda+\mu)} \phi_{t}(\mu)\right| \leq C \sum_{t}^{n}\left|\phi_{t}(\mu)\right| \leq C \sum_{t=1}^{n} t^{\alpha} \leq C n^{\alpha+1},
$$

while, using $\left|\phi_{t}(\lambda)\right| \leq C|\lambda|^{\alpha}$,

$$
\left|\phi_{n}(\lambda, \mu)\right| \leq C \sum_{t}^{n}|\mu|^{\alpha} \leq C n|\mu|^{\alpha} .
$$

Now, defining $D_{n}(x) \quad \sum_{t 1}^{n} \mathrm{e}^{\mathrm{i} t x}$ and using summation by parts,

$$
\phi_{n}(\lambda, \mu) \quad \phi_{n}(\mu) D_{n}(\lambda+\mu) \quad \sum_{t}^{n} \phi_{t}^{(\alpha)} \mathrm{e}^{\mathrm{i} t \mu} D_{t}(\lambda+\mu)
$$

[cf. (8.2) in Robinson and Marinucci (2001), which we are not able to reproduce here, and with (8.3), which is a straightforward consequence of our results]. Then, using that $\left|D_{n}(x)\right| \leq \min \left\{|x|^{1}, n\right\}$, we have that $\left|\phi_{n}(\mu) D_{n}(\lambda+\mu)\right| \leq C \min \left\{|\mu|^{\alpha}, n^{\alpha}\right\} \min$ $\left\{|\lambda+\mu|^{1}, n\right\}$. Similarly, we can obtain that

$$
\left|\sum_{t}^{n} \phi_{t}^{(\alpha)} \mathrm{e}^{\mathrm{i} t \mu} D_{t}(\lambda+\mu)\right| \leq C|\lambda+\mu|^{1} \sum_{t}^{n}\left|\phi_{t}^{(\alpha)}\right| \leq C|\lambda+\mu|^{1} \sum_{t}^{n} t^{\alpha}{ }^{1} \leq C n^{\alpha}|\lambda+\mu|^{1} .
$$

Finally, using that $D_{t}(x) \quad \mathrm{e}^{\mathrm{i} x}\left(1 \quad \mathrm{e}^{\mathrm{i} t x}\right) /\left(1 \quad \mathrm{e}^{\mathrm{i} x}\right)$ we have that the second term in eqn (15) is 


$$
\frac{\mathrm{e}^{\mathrm{i}(\lambda+\mu)}}{1 \quad \mathrm{e}^{\mathrm{i}(\lambda+\mu)}}\left\{\phi_{n}(\lambda) \quad \phi_{n}(\mu)\right\}
$$

and its modulus is bounded by $C|\lambda+\mu|^{1}\left\{|\mu|^{\alpha}+|\lambda|^{\alpha}\right\}$ and the lemma follows from this bound and eqns (13), (14) and (16).

QED

Lemma 2. Under Assumption 1, for $\left\{\phi_{t}^{(\alpha)}\right\} \in \Phi(\alpha), \quad 1<\alpha<0,\left|\phi_{t}(\lambda)\right| \leq C\left\{t^{\alpha}+|\lambda|{ }^{\alpha}\right\}$; $\left|\bar{\phi}_{t}(\lambda)\right| \leq C \min \left\{t^{\alpha}{ }^{1}|\lambda|^{1}, t^{\alpha}\right\}$; and $\left|\phi_{n}(\lambda, \mu)\right| \leq C\left\{n^{\alpha}+|\lambda|^{\alpha}+|\mu|^{\alpha}\right\} \min \left\{n,|\mu+\lambda|^{1}\right\}$.

Proof of Lemma 2. First we have for the bounds of $\left|\bar{\phi}_{t}(\lambda)\right|, \quad 1<\alpha<0$, that

$$
\bar{\phi}_{t}(\lambda) \quad \sum_{j t}^{\infty} \phi_{j}^{(\alpha)} \mathrm{e}^{\mathrm{i} t \lambda} \quad O\left(\sum_{j t}^{\infty} j^{\alpha} 1\right) \quad O\left(t^{\alpha}\right),
$$

while also

$$
\bar{\phi}_{t}(\lambda) \quad \sum_{j t}^{\infty}\left(\begin{array}{ll}
\phi_{j}^{(\alpha)} & \left.\phi_{j+1}^{(\alpha)}\right)
\end{array}\right) \sum_{a t}^{j} \mathrm{e}^{\mathrm{i} t \lambda} \quad O\left(\sum_{j t}^{\infty} j^{\alpha}{ }^{2}|\lambda|^{1}\right) \quad O\left(t^{\alpha}{ }^{1}|\lambda|^{1}\right) .
$$

Then, because $\phi(0) \quad \sum_{j 0}^{\infty} \phi_{j}^{(\alpha)} \quad 0$ we have that $\phi_{t}(0) \quad \sum_{j t}^{\infty} \phi_{j}^{(\alpha)} \quad O\left(t^{\alpha}\right)$, and also $\phi_{t}(\lambda) \quad \phi_{t}(0)+O\left(|\lambda|\left|\phi_{t}^{\prime}\left(\lambda^{*}\right)\right|\right)$ for some $\lambda^{*} \in[0, \lambda]$. Then, we can check that $\phi_{t}^{\prime}\left(\lambda^{*}\right) \quad O\left(\sum_{j}^{t} \begin{array}{ll}1 & j^{\alpha}\end{array}\right) \quad t^{\alpha+1}$, so $\phi_{t}(\lambda) \quad O\left(t^{\alpha}+|\lambda| t^{\alpha+1}\right) \quad t^{\alpha} O(1+|\lambda| t)$.

Therefore, if $|\lambda| \leq t^{1}$, then $\phi_{t}(\lambda) \quad O\left(t^{\alpha}\right)$, while if $|\lambda|>t^{1},\left|\phi_{t}(\lambda)\right| \leq|\phi(\lambda)|+$ $\left|\bar{\phi}_{t}(\lambda)\right| \leq C\left\{|\lambda|^{\alpha}+t^{\alpha}{ }^{1}|\lambda|^{1}\right\} \leq C|\lambda|^{\alpha}$, so $\left|\phi_{t}(\lambda)\right| \leq C\left\{t^{\alpha}+|\lambda|^{\alpha}\right\}$.

For the analysis of $\left|\phi_{n}(\lambda, \mu)\right|$ we can use eqns (15) and (17), with $\left|D_{t}(x)\right| \leq C|x|^{1}$, obtaining that

$$
\begin{aligned}
\left|\phi_{n}(\lambda, \mu)\right| & \leq C n^{\alpha}|\lambda+\mu|^{1}+C|\lambda+\mu|^{1}\left\{\left|\phi_{n}(\lambda)\right|+\left|\phi_{n}(\mu)\right|\right\} \\
& \leq C\left\{n^{\alpha}+|\mu|^{\alpha}\right\}|\lambda+\mu|^{1}+C|\lambda+\mu|^{1}\left\{n^{\alpha}+|\lambda|^{\alpha}+|\mu|^{\alpha}\right\} \\
& \leq C\left\{n^{\alpha}+|\lambda|^{\alpha}+|\mu|^{\alpha}\right\}|\lambda+\mu|^{1}
\end{aligned}
$$

and using $\left|D_{t}(x)\right| \leq C t$ and $\left|\phi_{t}(x)\right| \leq t^{\alpha}$,

$$
\left|\phi_{n}(\lambda, \mu)\right| \leq C\left\{n^{\alpha}+|\mu|^{\alpha}\right\} n+C \sum_{t}\left|\phi_{t}^{(\alpha)}\right| t \leq C\left\{n^{\alpha}+|\mu|^{\alpha}\right\} n .
$$

Proof of Theorem 1. We only prove the bounds for the first two expectations, since the proof for the rest is similar but simpler. We first deal with the case $\alpha>0$. Proceeding as in eqn (8.2) of Robinson and Marinucci (2001),

$$
\begin{aligned}
& \int_{\Pi} \chi_{j, k}(\mu) \mathrm{d} \mu \quad \sum_{t}^{n} \phi_{n}^{(\alpha)}{ }_{t} \mathrm{e}^{\mathrm{i}(n t) \lambda_{j}} \sum_{p} \phi_{n}^{(\alpha)}{ }_{p} \mathrm{e}^{\mathrm{i}(n \quad p) \lambda_{k}} D_{\min (t, p)}\left(\begin{array}{ll}
\lambda_{j} & \lambda_{k}
\end{array}\right) \\
& \left.2 \pi \sum_{t}^{n} \mathrm{e}^{\mathrm{i} t\left(\lambda_{j}\right.} \lambda_{k}\right) \sum_{a}^{n t} \phi_{a}^{(\alpha)} \mathrm{e}^{\mathrm{i} a \lambda_{j}} \sum_{b=0}^{n t} \phi_{b}^{(\alpha)} \mathrm{e}^{\mathrm{i} b \lambda_{k}}
\end{aligned}
$$

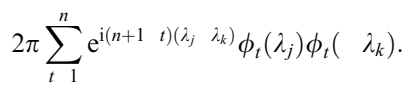


Then

$$
\begin{aligned}
E\left[w_{\zeta}\left(\lambda_{j}\right) w_{\zeta}\left(\lambda_{j}\right)\right] \quad f_{\zeta \zeta}\left(\lambda_{j}\right) \quad & \frac{1}{2 \pi n} \int_{\Pi} \chi_{j, j}(\mu)\left\{f_{\eta \eta}(\mu) \quad f_{\eta \eta}\left(\lambda_{j}\right)\right\} \mathrm{d} \mu \\
& +f_{\eta \eta}\left(\lambda_{j}\right)\left\{\frac{1}{n} \sum_{t=1}^{n}\left|\phi_{t}\left(\lambda_{j}\right)\right|^{2} \quad\left|\phi\left(\lambda_{j}\right)\right|^{2}\right\},
\end{aligned}
$$

because $\int_{\Pi} \chi_{j, j}(\mu) \mathrm{d} \mu \quad 2 \pi \sum_{t 1}^{n}\left|\phi_{t}\left(\lambda_{j}\right)\right|^{2}$. Writing

$$
|\phi(\lambda)|^{2} \quad\left|\phi_{t}(\lambda)\right|^{2} \quad \phi_{t}(\lambda) \bar{\phi}_{t}(\lambda)+\phi_{t}(\lambda) \bar{\phi}_{t}(\lambda)+\left|\bar{\phi}_{t}(\lambda)\right|^{2},
$$

we obtain from Lemma 1 for $\alpha>0$,

$$
\left.\left.|| \phi(\lambda)\right|^{2} \quad\left|\phi_{t}(\lambda)\right|^{2} \mid \leq C\left\{|\lambda|^{\alpha}{ }^{1} t^{\alpha} 1^{1}+|\lambda|^{2 \alpha} \min \left\{1,(t|\lambda|)^{2(\alpha} 1\right)\right\}\right\}
$$

(compare with discussion on p. 968 of Robinson and Marinucci, 2001) and therefore

$$
\begin{aligned}
& \left|\frac{1}{n} \sum_{t}\left\{\left|\phi_{t}\left(\lambda_{j}\right)\right|^{2} \quad\left|\phi\left(\lambda_{j}\right)\right|^{2}\right\}\right| \\
& \left.\leq \frac{C}{n} \sum_{t}\left|\lambda_{j}\right|^{2 \alpha}\left\{\left(\left|\lambda_{j}\right| t\right)^{\alpha} 1+\min \left\{1,\left(t\left|\lambda_{j}\right|\right)^{2(\alpha} 1\right)\right\}\right\} \\
& \left.\leq \frac{C}{n}\left|\lambda_{j}\right|^{2 \alpha}\left\{\left|\lambda_{j}\right|^{\alpha} 1 \sum_{t} t^{\alpha 1}+\sum_{t}^{\left|\lambda_{j}\right|^{1}} 1+\sum_{t\left|\lambda_{j}\right|}^{n}\left(t\left|\lambda_{j}\right|\right)^{2(\alpha} 1\right)\right\}
\end{aligned}
$$

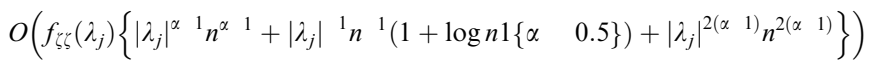

$$
\begin{aligned}
& O\left(f_{\zeta \zeta}\left(\lambda_{j}\right)\left\{\begin{array}{ll}
j^{\alpha}{ }^{1}+j^{1} \log n 1\{\alpha & 0.5
\end{array}\right\}\right) .
\end{aligned}
$$

In view of eqn (25), eqn (20) is $O\left(f_{\zeta \zeta}\left(\lambda_{j}\right)\left\{j^{\alpha}{ }^{1}+j^{1} \log n 1\{\alpha \quad 0.5\}\right\}\right), \alpha>0$

Fix one $\epsilon>0$ such that $f_{\eta \eta}(\lambda)$ is boundedly differentiable in the interval $(\epsilon, \epsilon)$. Then we can write eqn (19) as

$$
\frac{1}{2 \pi n} \int_{\pi}^{\pi} \chi_{j, j}\left(\lambda_{j}+\mu\right)\left\{f_{\eta \eta}\left(\lambda_{j}+\mu\right) \quad f_{\eta \eta}\left(\lambda_{j}\right)\right\} \mathrm{d} \mu,
$$

where $\quad \chi_{j, j}\left(\lambda_{j}+\mu\right) \quad \phi_{n}\left(\lambda_{j}, \quad \lambda_{j} \quad \mu\right) \phi_{n}\left(\lambda_{j}, \lambda_{j}+\mu\right), \quad$ and from Lemma 1 , $\left|\phi_{n}\left(\lambda_{j}, \quad \lambda_{j}, \mu\right)\right| \leq C L_{n}(\mu) \min \left\{n^{\alpha},\left[\left|\lambda_{j}+\mu\right|^{\alpha}+\left|\lambda_{j}\right|^{\alpha}\right]\right\}, \alpha>0$, where $L_{n}(\mu) \quad n,|\mu|<$ $n^{1} ; \quad|\mu|^{1},|\mu| \geq n^{1}$, so $\left|L_{n}(\mu)\right| \leq \min \left\{|\mu|^{1}, n\right\}$. Now we consider different intervals inside $[\pi, \pi]$ for the integral in eqn (26). First, we have that

$$
\begin{aligned}
\left|\int_{|\mu|<\lambda_{j} / 2}\right| & \leq C n^{1} \int_{|\mu|<\lambda_{j} / 2}\left|\phi_{n}\left(\lambda_{j}, \quad \lambda_{j} \quad \mu\right)\right|^{2}|\mu| \mathrm{d} \mu \\
& \leq C n^{1} \int_{|\mu|<\lambda_{j} / 2} \lambda_{j}{ }^{2 \alpha}\left|L_{n}(\mu)\right| \mathrm{d} \mu \quad O\left(f_{\zeta \zeta}\left(\lambda_{j}\right) n^{1} \log j\right),
\end{aligned}
$$

using that $\int_{|\mu|<\lambda_{j} / 2 \mid} L_{n}(\mu) \mid \mathrm{d} \mu \quad O(\log j)$, and 


$$
\begin{aligned}
\left|\int_{3 \lambda_{j} / 2}^{\lambda_{j} / 2}\right| & \leq C n^{1} \int_{3 \lambda_{j} / 2}^{\lambda_{j} / 2}\left|\phi_{n}\left(\lambda_{j}, \quad \lambda_{j} \quad \mu\right)\right|^{2}|\mu| \mathrm{d} \mu \\
& \leq C n^{1} \int_{3 \lambda_{j} / 2}^{\lambda_{j} / 2}|\mu|^{1}\left|\lambda_{j}+\mu\right|^{2 \alpha} \mathrm{d} \mu+C n^{1}\left|\lambda_{j}\right|^{2 \alpha} \int_{3 \lambda_{j} / 2}^{\lambda_{j} / 2}|\mu|^{1} \mathrm{~d} \mu \\
& \leq C n^{1} \lambda_{j}{ }^{1} \int_{\lambda_{j} / 2}^{\lambda_{j} / 2}|\mu|^{2 \alpha} \mathrm{d} \mu+C n^{1}\left|\lambda_{j}\right|{ }^{2 \alpha} \\
& \leq C n^{1} \lambda_{j}{ }^{2 \alpha} O\left(f_{\zeta \zeta}\left(\lambda_{j}\right) n{ }^{1}\right),
\end{aligned}
$$

when $\alpha<1 / 2$, while for $1 / 2<\alpha<1$ we obtain that

$$
\begin{aligned}
& \left|\int_{3 \lambda_{j} / 2}^{\lambda_{j} / 2}\right| \leq C n^{1} \int_{3 \lambda_{j} / 2}^{\lambda_{j} / 2}\left|\phi_{n}\left(\lambda_{j}, \quad \lambda_{j} \quad \mu\right)\right|^{2}|\mu| \mathrm{d} \mu \\
& \leq C n^{1} \int_{3 \lambda_{j} / 2}^{\lambda_{j} / 2} \min \left\{n^{2 \alpha},\left[\left|\lambda_{j}+\mu\right|^{2 \alpha}+\left|\lambda_{j}\right|{ }^{2 \alpha}\right]\right\}\left|L_{n}(\mu)\right|^{2}|\mu| \mathrm{d} \mu \\
& \leq C n{ }^{1} \lambda_{j}{ }^{1} \int_{\lambda_{j} / 2}^{\lambda_{j} / 2} \min \left\{n^{2 \alpha},|\mu|{ }^{2 \alpha}\right\} \mathrm{d} \mu+n{ }^{1} \lambda_{j}{ }^{1}\left|\lambda_{j}\right|^{12 \alpha} \\
& \leq C n{ }^{1} \lambda_{j}{ }^{1} n^{2 \alpha}{ }^{1}(\log j)^{\alpha}{ }^{0.5} \\
& \left.O\left(f_{\zeta \zeta}\left(\lambda_{j}\right) j^{2 \alpha}{ }^{1} n^{1}(\log j)^{\alpha} 0.5\right) \quad O\left(f_{\zeta \zeta}\left(\lambda_{j}\right) j^{2(\alpha} 1\right)(\log j)^{\alpha} \quad 0.5\right) .
\end{aligned}
$$

Next

$$
\begin{aligned}
\left|\int_{\lambda_{j} / 2}^{\epsilon}\right| \leq & C n^{1} \int_{\lambda_{j}}^{\epsilon}\left|\phi_{n}\left(\lambda_{j}, \quad \lambda_{j} \quad \mu\right)\right|^{2}|\mu| \mathrm{d} \mu \\
\leq & C n^{1} \int_{\lambda_{j}}^{\epsilon}\left\{\left|\lambda_{j}+\mu\right|^{2 \alpha}+\left|\lambda_{j}\right|^{2 \alpha}\right\}\left|L_{n}(\mu)\right| \mathrm{d} \mu \quad O\left(n^{1}{ }^{1} \lambda_{j}{ }^{2 \alpha} \log j\right) \\
& O\left(f_{\zeta \zeta}\left(\lambda_{j}\right) n^{1} \log j\right),
\end{aligned}
$$

while the contribution from the interval $\left[\begin{array}{ll}\epsilon & 3 \lambda_{j} / 2\end{array}\right]$ can be dealt with similarly.

Finally, using the integrability of $f_{\eta \eta}(\mu)$,

$$
\begin{aligned}
\left|\int_{\epsilon \leq|\mu| \leq \pi}\right| \leq & C n^{1} \int_{\epsilon \leq|\mu| \leq \pi}\left|\phi_{n}\left(\lambda_{j}, \quad \lambda_{j} \quad \mu\right)\right|^{2}\left\{f_{\eta \eta}\left(\lambda_{j}+\mu\right)+f_{\eta \eta}\left(\lambda_{j}\right)\right\} \mathrm{d} \mu \\
\leq & C n^{1} \int_{\epsilon \leq|\mu| \leq \pi}\left(\left|\lambda_{j}+\mu\right|^{2 \alpha}+\left|\lambda_{j}\right|^{2 \alpha}\right)|\mu|^{2}\left\{f_{\eta \eta}\left(\lambda_{j}+\mu\right)+f_{\eta \eta}\left(\lambda_{j}\right)\right\} \mathrm{d} \mu \\
& n^{1}\left|\lambda_{j}\right|^{2 \alpha} O\left(1+\int_{\epsilon \leq|\mu| \leq \pi} f_{\eta \eta}\left(\lambda_{j}+\mu\right) \mathrm{d} \mu\right) \quad O\left(f_{\zeta \zeta}\left(\lambda_{j}\right) n{ }^{1}\right) .
\end{aligned}
$$

Now to bound $E\left[w_{\zeta}\left(\lambda_{j}\right) w_{\zeta}\left(\lambda_{k}\right)\right] \quad(2 \pi n){ }^{1} \int_{\Pi} \chi_{j, k}(\mu) f_{\eta \eta}(\mu) \mathrm{d} \mu$ we distinguish two cases, values of $j$ and $k$ close and far away. If $j / 2 \leq k<j$, using that $\left.\sum_{t} \mathrm{e}^{\mathrm{i}(n+1} \quad t\right)\left(\lambda_{j} \quad \lambda_{k}\right)$ $\sum_{t} e^{\mathrm{i} t\left(\lambda_{j}\right.} \lambda_{\left.\lambda_{k}\right)} \quad 0, j / k(\bmod n),(2 \pi){ }^{1} \int_{\Pi} \chi_{j, k}(\mu) \mathrm{d} \mu$ is by eqn (18) equal to 


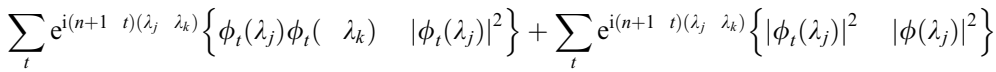

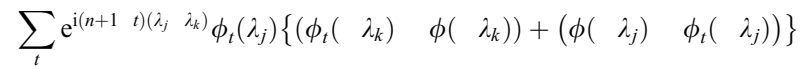

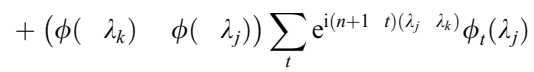

$$
\begin{aligned}
& \left.+\sum_{t} \mathrm{e}^{\mathrm{i}(n+1} \quad t\right)\left(\lambda_{j} \lambda_{k}\right)\left\{\left|\phi_{t}\left(\lambda_{j}\right)\right|^{2} \quad\left|\phi\left(\lambda_{j}\right)\right|^{2}\right\}
\end{aligned}
$$

Using $|(\mathrm{d} / \mathrm{d} \lambda) \phi(\lambda)| \leq C|\lambda|^{\alpha{ }^{1}}$ as $|\lambda| \rightarrow 0$, as $k^{1} \quad O\left(j^{1}\right), j / 2 \leq k<j$, and the mean value theorem (MVT),

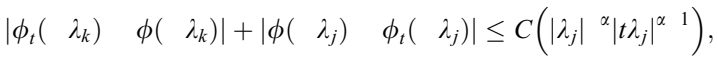

$$
\begin{aligned}
& \left|\phi\left(\lambda_{k}\right) \quad \phi\left(\lambda_{j}\right)\right| \leq C\left|\lambda_{j}\right|^{\alpha 1}{ }^{1} \lambda_{j} \quad \lambda_{k} \mid,
\end{aligned}
$$

while using summation by parts

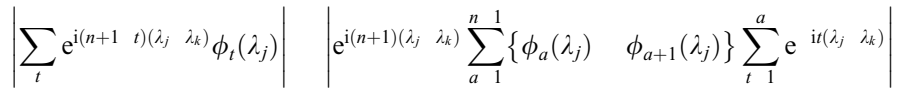

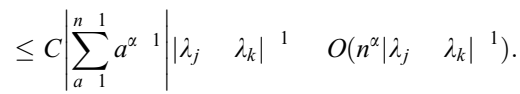

Then, as $k^{1} \quad O\left(j^{1}\right), j / 2 \leq k<j$, and using eqn (22), we obtain

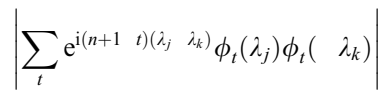

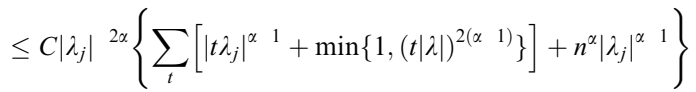

$$
\begin{aligned}
& \leq C n\left|\lambda_{j}\right|^{2 \alpha}\left[n^{\alpha}{ }^{1}\left|\lambda_{j}\right|^{\alpha 1}+n^{1}\left|\lambda_{j}\right|^{1}\left(1+\log n 1_{0.5}\right)\right] \\
& O\left(n \sqrt{f_{\zeta \zeta}}\left(\lambda_{j}\right) f_{\zeta \zeta}\left(\lambda_{k}\right)\left\{j^{\alpha}{ }^{1}+j^{1} \log n 1_{0.5}\right\}\right) .
\end{aligned}
$$

When $1 \leq k<j / 2$ then $|j \quad k|^{1} \leq 2 j^{1}$, and using summation by parts,

$$
\begin{aligned}
\frac{1}{2 \pi} \int_{\Pi} \chi_{j, k}(\mu) \mathrm{d} \mu & \left.\sum_{t=1}^{n}\left\{\phi_{t}\left(\lambda_{j}\right) \phi_{t}\left(\lambda_{k}\right) \quad \phi_{t+1}\left(\lambda_{j}\right) \phi_{t+1}\left(\lambda_{k}\right)\right\} \sum_{a}^{t} \mathrm{e}^{\mathrm{i}\left(\begin{array}{lll}
n+1 & a
\end{array}\right)\left(\lambda_{j}\right.} \lambda_{k}\right) \\
& +\phi_{n}\left(\lambda_{j}\right) \phi_{n}\left(\lambda_{k}\right) \sum_{t} \mathrm{e}^{\mathrm{i}\left(\begin{array}{lll}
n+1 & t
\end{array}\right)\left(\lambda_{j} \lambda_{k}\right)} .
\end{aligned}
$$

Now eqn (31) is exactly zero when $j / k(\bmod n)$, and with

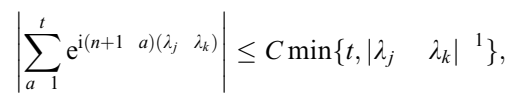

the modulus of the right hand side of eqn (30) is bounded by 


$$
\begin{aligned}
& C \sum_{t}^{n}\left\{\left|\phi_{t}\left(\lambda_{j}\right)\right|\left|\phi_{t}\left(\lambda_{k}\right) \quad \phi_{t+1}\left(\quad \lambda_{k}\right)\right|+\left|\phi_{t+1}\left(\quad \lambda_{k}\right)\right|\left|\phi_{t}\left(\lambda_{j}\right) \quad \phi_{t+1}\left(\lambda_{j}\right)\right|\right\}\left|\lambda_{j} \quad \lambda_{k}\right|^{1} \\
& \leq C \sum_{t}^{n}\left|\phi_{t}\right|\left\{\left|\phi_{t}\left(\lambda_{j}\right)\right|+\left|\phi_{t}\left(\lambda_{k}\right)\right|\right\}\left|\lambda_{j} \quad \lambda_{k}\right|^{1} \\
& O\left(\left|\phi_{t}\left(\lambda_{k}\right)\right|\left|\lambda_{j} \quad \lambda_{k}\right|^{1} \sum_{t}^{n} t^{\alpha} t^{1}\right) \quad O\left(\left|\lambda_{k} \lambda_{j}\right|^{\alpha}|j \quad k|^{1}\left|\lambda_{j}\right|^{\alpha} n^{\alpha+1}\right) \\
& O\left(n \sqrt{f_{\zeta \zeta}}\left(\lambda_{j}\right) f_{\zeta \zeta}\left(\lambda_{k}\right) j^{\alpha} 1\right) .
\end{aligned}
$$

Therefore, from eqns (29) and (32) we obtain that, $1 \leq k<j<n / 2$,

$$
\frac{1}{2 \pi n} \int_{\Pi} \chi_{j, k}(\mu) \mathrm{d} \mu \quad O\left(\sqrt{f_{\zeta \zeta}}\left(\lambda_{j}\right) f_{\zeta \zeta}\left(\lambda_{k}\right)\left\{j^{\alpha}{ }^{1}+j^{1} \log n 1_{0.5}\right\}\right) .
$$

Next, with $\quad \chi_{j, k}(\mu) \quad \phi_{n}\left(\lambda_{j}, \quad \mu\right) \phi_{n}\left(\lambda_{k}, \mu\right), \quad\left|\phi_{n}\left(\lambda_{j}, \quad \mu\right)\right| \leq C\left|L_{n}\left(\lambda_{j} \quad \mu\right)\right|$ $\min \left\{n^{\alpha},\left(|\mu|^{\alpha}+\lambda_{j}^{\alpha}\right)\right\}$, following Robinson (1995a, p. 1063), we obtain that

$$
\begin{aligned}
E\left[w_{\zeta}\left(\lambda_{j}\right) w_{\zeta}\left(\lambda_{k}\right)\right] \quad & \frac{1}{2 \pi n} \int_{\left(\lambda_{j}+\lambda_{k}\right) / 2}^{2 \lambda_{j}} \chi_{j, k}(\mu)\left\{f_{\eta \eta}(\mu) \quad f_{\eta \eta}\left(\lambda_{j}\right)\right\} \mathrm{d} \mu \\
& +\frac{1}{2 \pi n} \int_{\lambda_{k} / 2}^{\left(\lambda_{j}+\lambda_{k}\right) / 2} \chi_{j, k}(\mu)\left\{f_{\eta \eta}(\mu) \quad f_{\eta \eta}\left(\lambda_{k}\right)\right\} \mathrm{d} \mu \\
& \frac{1}{2 \pi n}\left\{f_{\eta \eta}\left(\lambda_{j}\right) \quad f_{\eta \eta}\left(\lambda_{k}\right)\right\} \int_{\lambda_{k} / 2}^{\left(\lambda_{j}+\lambda_{k}\right) / 2} \chi_{j, k}(\mu) \mathrm{d} \mu \\
& +\frac{1}{2 \pi n}\left\{\int_{2 \lambda_{j}}^{\pi}+\int_{\pi}^{\lambda_{k} / 2}\right\} \chi_{j, k}(\mu)\left\{f_{\eta \eta}(\mu) \quad f_{\eta \eta}\left(\lambda_{j}\right)\right\} \mathrm{d} \mu .
\end{aligned}
$$

Now using the differentiability of $f_{\eta \eta}(\mu)$ in $\mu \in(\epsilon, \epsilon)$, eqn (33) is bounded by

$$
\begin{aligned}
& \frac{1}{2 \pi n} \sup _{\left(\lambda_{j}+\lambda_{k}\right) / 2 \leq \mu \leq 2 \lambda_{j}}\left|f_{\eta \eta}^{\prime}(\mu)\right| \int_{\left(\lambda_{j}+\lambda_{k}\right) / 2}^{2 \lambda_{j}}\left|\phi_{n}\left(\lambda_{j}, \quad \mu\right) \phi_{n}\left(\lambda_{k}, \mu\right)\right|\left|\lambda_{j} \quad \mu\right| \mathrm{d} \mu \\
& \leq C\left(\lambda_{j} \lambda_{k}\right){ }^{\alpha}{ }_{n}{ }^{1} \int_{\left(\lambda_{j}+\lambda_{k}\right) / 2}^{2 \lambda_{j}}\left|L_{n}\left(\lambda_{k} \quad \mu\right)\right| \mathrm{d} \mu \quad O\left(\left(\lambda_{j} \lambda_{k}\right){ }^{\alpha}{ }_{n}{ }^{1} \log j\right) .
\end{aligned}
$$

Next eqn (34) is bounded by

$$
\frac{C \lambda_{k}^{2 \alpha}}{2 \pi n} \sup _{\lambda_{k} / 2 \leq \mu \leq\left(\lambda_{j}+\lambda_{k}\right) / 2}\left|f_{\eta \eta}^{\prime}(\mu)\right| \int_{\lambda_{k} / 2}^{\left(\lambda_{j}+\lambda_{k}\right) / 2}\left|L_{n}\left(\lambda_{j} \quad \mu\right)\right| \mathrm{d} \mu \quad O\left(\lambda_{k}{ }^{2 \alpha} n^{1} \log j\right) .
$$

Now eqn (35) is bounded by

$$
\begin{array}{r}
\frac{1}{2 \pi n}\left(\begin{array}{ll}
\lambda_{j} & \lambda_{k}
\end{array}\right) \sup _{\lambda_{k} \leq \mu \leq \lambda_{j}}\left|f_{\eta \eta}^{\prime}(\mu)\right| \int_{\lambda_{k} / 2}^{\left(\lambda_{j}+\lambda_{k}\right) / 2}\left|\phi_{n}\left(\lambda_{j}, \quad \mu\right) \phi_{n}\left(\quad \lambda_{k}, \mu\right)\right| \mathrm{d} \mu \\
O\left(n^{1} \lambda_{k}{ }^{2 \alpha} \int_{\lambda_{k} / 2}^{\left(\lambda_{j}+\lambda_{k}\right) / 2} \mid L_{n}\left(\begin{array}{ll}
\mu & \left.\left.\lambda_{k}\right) \mid \mathrm{d} \mu\right)
\end{array}\right) \quad O\left(\lambda_{k}{ }^{2 \alpha} n^{1} \log j\right) .\right.
\end{array}
$$

Finally, consider eqn (36). If $\alpha<0.5$, then 


$$
\begin{aligned}
& \frac{1}{2 \pi n} \int_{\lambda_{k} / 2}^{\lambda_{k} / 2}\left|\phi_{n}\left(\lambda_{j}, \quad \mu\right) \phi_{n}\left(\lambda_{k}, \mu\right)\right|\left\{f_{\eta \eta}(\mu) \quad f_{\eta \eta}\left(\lambda_{j}\right)\right\} \mathrm{d} \mu \\
& \leq C n^{1} \sup _{\lambda_{k} / 2 \leq \mu \leq \lambda_{k} / 2}\left|f_{\eta \eta}^{\prime}(\mu)\right| \int_{\lambda_{k} / 2}^{\lambda_{k} / 2}\left|L_{n}\left(\lambda_{k} \quad \mu\right)\right|\left(|\mu|^{\alpha}+\left|\lambda_{k}\right|^{\alpha}\right)\left(|\mu|^{\alpha}+\left|\lambda_{j}\right|^{\alpha}\right) \mathrm{d} \mu \\
& \leq C n^{1} \lambda_{k}{ }^{1}\left\{\left|\lambda_{k}\right|^{12 \alpha}+\left|\lambda_{k}\right|^{1}{ }^{\alpha}\left|\lambda_{j}\right|^{\alpha}\right\} \leq C \lambda_{k}{ }^{2 \alpha} n^{1},
\end{aligned}
$$

and if $\alpha \geq 0.5$

$$
\begin{aligned}
& \frac{1}{2 \pi n} \int_{\lambda_{k} / 2}^{\lambda_{k} / 2}\left|\phi_{n}\left(\lambda_{j}, \quad \mu\right) \phi_{n}\left(\lambda_{k}, \mu\right)\right|\left\{f_{\eta \eta}(\mu) \quad f_{\eta \eta}\left(\lambda_{j}\right)\right\} \mathrm{d} \mu \\
& \leq C n^{1} \sup _{\lambda_{k} / 2 \leq \mu \leq \lambda_{k} / 2}\left|f_{\eta \eta}^{\prime}(\mu)\right| \int_{\lambda_{k} / 2}^{\lambda_{k} / 2}\left|L_{n}\left(\lambda_{k} \quad \mu\right)\right|\left\{n^{\alpha} \wedge\left(|\mu|^{\alpha}+\left|\lambda_{k}\right|^{\alpha}\right)\right\} \\
& \quad \times\left\{n^{\alpha} \wedge\left(|\mu|^{\alpha}+\left|\lambda_{j}\right|^{\alpha}\right)\right\} \mathrm{d} \mu \\
& \leq C n^{1} \lambda_{k}{ }^{1}\left\{n^{2 \alpha} 1^{1}(\log j)^{\alpha} 0.5+\left|\lambda_{k}\right|^{1}{ }^{\alpha}\left|\lambda_{k}\right|^{\alpha}\right\} \leq C \lambda_{k}{ }^{2 \alpha} \lambda_{k}^{2 \alpha}{ }^{1} n^{2(\alpha} 1^{1)}(\log j)^{\alpha} 0.5 \\
& \leq C\left(\lambda_{k} \lambda_{j}\right)^{\alpha} k^{\alpha}{ }^{1} j^{\alpha} n^{1}(\log j)^{\alpha}{ }^{0.5} \leq C\left(\lambda_{k} \lambda_{j}\right)^{\alpha} j^{\alpha} 1 .
\end{aligned}
$$

The bounds for the remaining intervals follow now in a simpler way. Then using

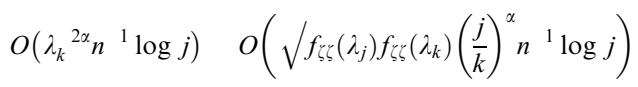

the theorem follows for $\alpha>0$.

We now deal with the case $\alpha<0$. Following the proof for $\alpha>0$, using eqn (21) and Lemma 2 now, we have that eqn (23) is bounded by, $0.5<\alpha<0$,

$$
\begin{aligned}
& \frac{C}{n}\left\{\sum_{t}^{\left|\lambda_{j}\right|^{1}} t^{2 \alpha}+\sum_{t}^{n} t^{2 \alpha}{ }^{2 \alpha}{ }^{1}\left|\lambda_{j}\right|^{1}+\sum_{t}^{\left|\lambda_{j}\right|^{1}} t^{2 \alpha}+\sum_{t\left|\lambda_{j}\right|}^{n} t^{2 \alpha}{ }^{1}\left|\lambda_{j}\right|^{1}\right\} \\
& O\left(n^{1}\left|\lambda_{j}\right|^{2 \alpha}{ }^{1}\right) \quad O\left(f_{\zeta \zeta}\left(\lambda_{j}\right) j^{1}\right) .
\end{aligned}
$$

and if $1<\alpha \leq 0.5$, we obtain that eqn (23) is

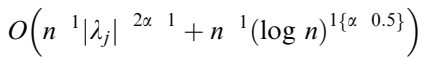

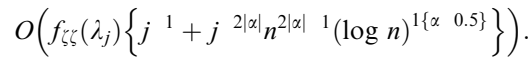

We have to bound also the following integrals,

$$
\frac{1}{2 \pi n}\left[\int_{|\mu|<n \mid}+\int_{n{ }^{1} \leq|\mu|<2 \lambda_{j}}++\int_{2 \lambda_{j} \leq|\mu|<\epsilon}+\int_{\epsilon \leq|\mu| \leq \pi}\right] \chi_{j, j}\left(\lambda_{j}+\mu\right)\left\{f_{\eta \eta}\left(\lambda_{j}+\mu\right) \quad f_{\eta \eta}\left(\lambda_{j}\right)\right\} \mathrm{d} \mu .
$$

Now $\quad \chi_{j, j}\left(\mu+\lambda_{j}\right) \quad \phi_{n}\left(\lambda_{j}, \quad \lambda_{j} \quad \mu\right) \phi_{n}\left(\lambda_{j}, \lambda_{j}+\mu\right), \quad\left|\phi_{n}\left(\lambda_{j}, \quad \lambda_{j} \quad \mu\right)\right| \leq C\left\{\left|\lambda_{j}\right|^{\alpha}+\right.$ $\left.\left|\lambda_{j}+\mu\right|^{\alpha}\right\} L_{n}(\mu)$, so

$$
\begin{aligned}
\left|\int_{|\mu|<n<1}\right| & \leq C n^{1} \int_{|\mu|<n=1}\left|\phi_{n}\left(\lambda_{j}, \quad \lambda_{j} \quad \mu\right)\right|^{2}|\mu| \mathrm{d} \mu \\
& \leq C \lambda_{j}{ }^{2 \alpha} n \int_{|\mu|<n_{1}}|\mu| \mathrm{d} \mu \quad O\left(f_{\zeta \zeta}\left(\lambda_{j}\right) n^{1}\right),
\end{aligned}
$$




$$
\begin{aligned}
\left|\int_{n}{ }^{1} \leq\right| \mu\left|<2 \lambda_{j}\right| & \leq C n^{1} \int_{n^{1} \leq|\mu|<2 \lambda_{j}}\left|\phi_{n}\left(\lambda_{j}, \quad \lambda_{j} \quad \mu\right)\right|^{2}|\mu| \mathrm{d} \mu \\
& \leq C n^{1} \lambda_{j}{ }^{2 \alpha} \int_{n^{1} \leq|\mu|<2 \lambda_{j}} L_{n}(\mu) \mathrm{d} \mu \quad O\left(f_{\zeta \zeta}\left(\lambda_{j}\right) n^{1} \log j\right), \\
\left|\int_{2 \lambda_{j} \leq|\mu|<\epsilon}\right| \leq C n^{1} \int_{2 \lambda_{j} \leq|\mu|<\epsilon} \mid \phi_{n}\left(\lambda_{j}, \quad \lambda_{j}\right. & \mu)\left.\right|^{2}|\mu| \mathrm{d} \mu \\
& \leq C n^{1} \int_{2 \lambda_{j} \leq|\mu|<\epsilon} L_{n}(\mu) \mathrm{d} \mu \quad O\left(n^{1}\right) \quad O\left(f_{\zeta \zeta}\left(\lambda_{j}\right) j^{2|\alpha|} n^{2|\alpha|} 1\right),
\end{aligned}
$$

and finally, using the integrability of $f_{\eta \eta}(\mu)$,

$$
\begin{aligned}
\left|\int_{\epsilon \leq|\mu| \leq \pi}\right| \leq & C n^{1} \int_{\epsilon \leq|\mu| \leq \pi}\left|\phi_{n}\left(\lambda_{j}, \quad \lambda_{j} \quad \mu\right)\right|^{2}\left\{f_{\eta \eta}\left(\lambda_{j}+\mu\right)+f_{\eta \eta}\left(\lambda_{j}\right)\right\} \mathrm{d} \mu \\
\leq & C n^{1} \int_{\epsilon \leq|\mu| \leq \pi}|\mu|^{2}\left\{f_{\eta \eta}\left(\lambda_{j}+\mu\right)+f_{\eta \eta}\left(\lambda_{j}\right)\right\} \mathrm{d} \mu \\
& n^{1} O\left(1+\int_{\epsilon \leq|\mu| \leq \pi} f_{\eta \eta}\left(\lambda_{j}+\mu\right) \mathrm{d} \mu\right) \quad O\left(f_{\zeta \zeta}\left(\lambda_{j}\right) j^{2|\alpha|} n^{2|\alpha|} 1\right) .
\end{aligned}
$$

For the proof of the second statement of the theorem, we have that $E\left[w_{\zeta}\left(\lambda_{j}\right) w_{\zeta}\left(\lambda_{k}\right)\right]$ $(2 \pi n){ }^{1} \int_{\Pi} \chi_{j, k}(\mu) f_{\eta \eta}(\mu) \mathrm{d} \mu$. Consider first $j / 2 \leq k<j$. Using $|(d / d \lambda) \phi(\lambda)| \leq\left. C|\lambda|\right|^{\alpha}{ }^{1}$ as $|\lambda| \rightarrow 0$, as $k^{1} O\left(j^{1}\right), j / 2 \leq k<j$, and the MVT,

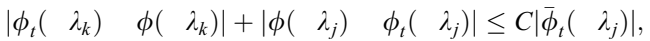

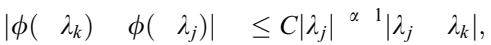

while by summation by parts

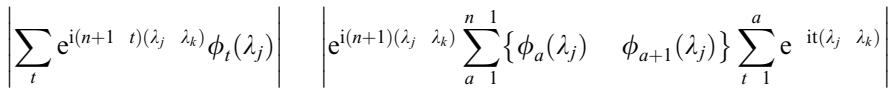

$$
\begin{aligned}
& \leq C\left|\sum_{a 1}^{\mid \lambda_{j}} \lambda_{k}\right|^{1} a^{\alpha}|+C| \sum_{\left|\lambda_{j} \lambda_{\lambda_{k}}\right|^{1}+1}^{n} a^{\alpha} 1 \mid \begin{array}{ll}
\mid \lambda_{j} & \left.\lambda_{k}\right|^{1}
\end{array} \\
& O\left(\left.\begin{array}{lll}
\mid \lambda_{j} & \lambda_{k}
\end{array}\right|^{\alpha}{ }^{1}\right) \text {. }
\end{aligned}
$$

Then, from eqn (27), because $k^{1} \quad O\left(j^{1}\right), j / 2 \leq k<j$, and following the methods used to obtain (37) and (38),

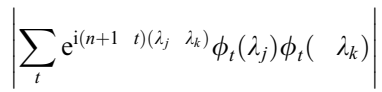

$$
\begin{aligned}
& \leq C \sum_{t}\left|\phi_{t}\left(\lambda_{j}\right)\right|\left|\bar{\phi}_{t}\left(\lambda_{j}\right)\right|+\left|\lambda_{j}\right|^{2 \alpha}{ }^{1}+\left.\sum_{t}|| \phi_{t}\left(\lambda_{j}\right)\right|^{2} \quad\left|\bar{\phi}_{t}\left(\lambda_{j}\right)\right|^{2} \mid \\
& O\left(n f_{\zeta \zeta}\left(\lambda_{j}\right)\left\{j^{1}+j^{2|\alpha|} n^{2|\alpha|}{ }^{1} \log n 1(\alpha \quad 0.5)\right\}\right) .
\end{aligned}
$$


When $1 \leq k<j / 2$ then $|j \quad k|^{1} \leq 2 j^{1}$, and using summation by parts as in eqns (30) and (31), we have that eqn (31) is zero when $j / k(\bmod n)$, and using Lemma 2 the modulus of eqn (30) is bounded by

$$
\begin{aligned}
& C \sum_{t=1}^{n}\left\{\left|\phi_{t}\left(\lambda_{j}\right)\right|\left|\phi_{t}\left(\lambda_{k}\right) \quad \phi_{t+1}\left(\lambda_{k}\right)\right|+\left|\phi_{t+1}\left(\quad \lambda_{k}\right)\right|\left|\phi_{t}\left(\lambda_{j}\right) \quad \phi_{t+1}\left(\lambda_{j}\right)\right|\right\}\left\{\left|\lambda_{j} \quad \lambda_{k}\right|^{1} \wedge t\right\} \\
& \leq C \sum_{t}^{\left|\lambda_{j}\right|^{1}}\left|\phi_{t}\right|\left\{\left|\phi_{t}\left(\lambda_{j}\right)\right|+\left|\phi_{t}\left(\lambda_{k}\right)\right|\right\} t+\sum_{t\left|\lambda_{j}\right|^{1}+1}^{n}\left|\phi_{t}\right|\left\{\left|\phi_{t}\left(\lambda_{j}\right)\right|+\left|\phi_{t}\left(\lambda_{k}\right)\right|\right\}\left|\lambda_{j} \quad \lambda_{k}\right|^{1} \\
& \leq C \sum_{t}^{\left|\lambda_{j}\right|^{1}} t^{\alpha}{ }^{1} t^{\alpha} t+C\left|\lambda_{j}\right|^{1} \sum_{\left.t \lambda_{j}\right|^{1}+1}^{n} t^{\alpha}{ }^{1}\left\{\left|\phi_{t}\left(\lambda_{j}\right)\right|+\left|\phi_{t}\left(\lambda_{k}\right)\right|\right\} \\
& \leq C\left|\lambda_{j}\right|^{12 \alpha}+C \log n 1(\alpha \quad 0.5)+C\left|\lambda_{j}\right|^{2 \alpha} \\
& O\left(n f_{\zeta \zeta}\left(\lambda_{j}\right)\left\{j^{1}+j^{2|\alpha|} n^{2|\alpha|}{ }^{1} \log n 1(\alpha \quad 0.5)\right\}\right) .
\end{aligned}
$$

Then, $\chi_{j, k}(\mu) \quad \phi_{n}\left(\lambda_{j}, \quad \mu\right) \phi_{n}\left(\lambda_{k}, \mu\right), \quad\left|\phi_{n}\left(\lambda_{j}, \mu\right)\right| \leq C\left\{\left|\lambda_{j}\right|^{\alpha}+|\mu|^{\alpha}\right\} \min \left\{\left|\lambda_{j}+\mu\right|^{1}, n\right\}$, $\alpha<0$, so following Robinson (1995a, p. 1063), we are led to estimate the contribution of eqns (33) (36) when $1<\alpha<0$. Using the differentiability of $f_{\eta \eta}(\mu)$ in $\mu \in(\epsilon, \epsilon)$, eqn (33) is bounded by

$$
\begin{aligned}
& \frac{1}{2 \pi n} \sup _{\left(\lambda_{j}+\lambda_{k}\right) / 2 \leq \mu \leq 2 \lambda_{j}}\left|f_{\eta \eta}^{\prime}(\mu)\right| \int_{\left(\lambda_{j}+\lambda_{k}\right) / 2}^{2 \lambda_{j}}\left|\phi_{n}\left(\lambda_{j}, \quad \mu\right) \phi_{n}\left(\lambda_{k}, \mu\right)\right|\left|\lambda_{j} \quad \mu\right| \mathrm{d} \mu \\
& \quad \leq C \lambda_{j}{ }^{2 \alpha} n^{1} \int_{\left(\lambda_{j}+\lambda_{k}\right) / 2}^{2 \lambda_{j}}\left|L_{n}\left(\mu \quad \lambda_{k}\right)\right| \mathrm{d} \mu \quad O\left(\lambda_{j}{ }^{2 \alpha} n^{1} \log j\right) .
\end{aligned}
$$

Next eqn (34) is bounded by

$$
\begin{array}{cc}
\frac{C \lambda_{j}{ }^{\alpha}}{2 \pi n} \sup _{\lambda_{k} / 2 \leq \mu \leq\left(\lambda_{j}+\lambda_{k}\right) / 2}\left|f_{\eta \eta}^{\prime}(\mu)\right| \int_{\lambda_{k} / 2}^{\left(\lambda_{j}+\lambda_{k}\right) / 2}\left|\phi_{n}\left(\lambda_{j}, \quad \mu\right)\right| \mathrm{d} \mu & O\left(\lambda_{j}{ }^{2 \alpha} n^{1} \int_{\lambda_{k}}^{\lambda_{j}} L_{n}(\mu) \mathrm{d} \mu\right) \\
& O\left(\lambda_{j}{ }^{2 \alpha} n^{1} \log j\right),
\end{array}
$$

and eqn (35) by

$$
\begin{array}{r}
\frac{1}{2 \pi n}\left(\begin{array}{ll}
\lambda_{j} & \left.\lambda_{k}\right)
\end{array} \sup _{\lambda_{k} \leq \mu \leq \lambda_{j}}\left|f_{\eta \eta}^{\prime}(\mu)\right| \int_{\lambda_{k} / 2}^{\left(\lambda_{j}+\lambda_{k}\right) / 2}\left|\phi_{n}\left(\lambda_{j}, \quad \mu\right) \phi_{n}\left(\lambda_{k}, \mu\right)\right| \mathrm{d} \mu\right. \\
O\left(n^{1} \lambda_{j}{ }^{\alpha} \int_{\lambda_{k} / 2}^{\left(\lambda_{j}+\lambda_{k}\right) / 2}\left|\phi_{n}\left(\lambda_{k}, \mu\right)\right| \mathrm{d} \mu\right) \quad O\left(\lambda_{j}{ }^{2 \alpha} n^{1} \log j\right) .
\end{array}
$$

Finally, eqn (36) is, using the integrability of $f_{\eta \eta}(\mu)$,

$$
\begin{aligned}
& \frac{1}{2 \pi n}\left\{\int_{2 \lambda_{j}}^{\pi}+\int_{\pi}^{\lambda_{k} / 2}\right\}\left|\phi_{n}\left(\lambda_{j}, \quad \mu\right) \phi_{n}\left(\lambda_{k}, \mu\right)\right|\left\{f_{\eta \eta}(\mu) \quad f_{\eta \eta}\left(\lambda_{j}\right)\right\} \mathrm{d} \mu \\
& \quad \leq \frac{C}{2 \pi n}\left\{\int_{2 \lambda_{j}}^{\pi}+\int_{\pi}^{\lambda_{k} / 2}\right\} f_{\eta \eta}(\mu) \mathrm{d} \mu \quad O\left(n^{1}\right) .
\end{aligned}
$$


Then using $O\left(n^{1}\right) \quad O\left(\sqrt{ } f_{\zeta \zeta}\left(\lambda_{j}\right) f_{\zeta \zeta}\left(\lambda_{k}\right)(k j){ }^{|\alpha|} n^{2|\alpha|} 1\right) \quad$ and $O\left(\lambda_{j}{ }^{2 \alpha} n^{1}\right)$ $\sqrt{ } f_{\zeta \zeta}\left(\lambda_{j}\right) f_{\zeta \zeta}\left(\lambda_{k}\right) O\left((j / n)^{2|\alpha|}(k j)^{|\alpha|} n^{2|\alpha|} 1\right) \quad O\left(\sqrt{ } f_{\zeta \zeta}\left(\lambda_{j}\right) f_{\zeta \zeta}\left(\lambda_{k}\right)(k j){ }^{|\alpha|} n^{2|\alpha|} 1\right)$ the theorem follows.

QED

Proof of Corollary 1. It is enough to show that Lemma 1 holds. The proof of Lemma 2 is similar. To show that the approximations of $\phi_{t+T+1}(\lambda)$ by $\phi(\lambda)$ have the same bounds as when $T \quad 1$, we have now that

$$
\left.\int_{\Pi} \chi_{j, k}(\mu) \mathrm{d} \mu \quad 2 \pi \sum_{t} \mathrm{e}^{\mathrm{i}(n+1)\left(\lambda_{j}\right.} \lambda_{k}\right) \phi_{t+T+1}\left(\lambda_{j}\right) \phi_{t+T+1}\left(\lambda_{k}\right) .
$$

Then $\left|\phi_{t+T+1}(\lambda)\right| \leq C \min \left\{|\lambda|^{\alpha},(T+t)^{\alpha}\right\}$ and $\left|\bar{\phi}_{T+t+1}(\lambda)\right| \leq C|\lambda|^{1}(T+t)^{\alpha}{ }^{1} \leq C^{\prime}|\lambda|^{1}$ $t^{\alpha}{ }^{1},\left|\bar{\phi}_{T+t+1}(\lambda)\right| \leq C|\lambda|^{\alpha}, \alpha>0$, so we can obtain

$$
|\phi(\lambda)|^{2} \quad\left|\phi_{t+T+1}(\lambda)\right|^{2} \quad \phi_{t+T+1}(\lambda) \bar{\phi}_{T+t+1}(\lambda)+\phi_{t+T+1}(\lambda) \bar{\phi}_{T+t+1}(\lambda)+\left|\bar{\phi}_{t+T+1}(\lambda)\right|^{2}
$$

and that, $0<\alpha<1$,

$$
\begin{aligned}
& \left.\left.|| \phi(\lambda)\right|^{2} \quad\left|\phi_{t+T+1}(\lambda)\right|^{2}|\leq C| \lambda \mid{ }^{2 \alpha}\left\{[|\lambda|(T+t)]^{\alpha}{ }^{1} \min \left\{1,((T+t)|\lambda|)^{2(\alpha} 1\right)\right\}\right\} \\
& \left.\leq C|\lambda|^{2 \alpha}\left\{[|\lambda| t]^{\alpha}{ }^{1}+[|\lambda| t]^{2(\alpha} 1\right)\right\} .
\end{aligned}
$$

Then $\phi_{n, T}\left(\lambda_{j}, \mu\right) \quad \sum_{t} \mathrm{e}^{\mathrm{i} t\left(\lambda_{j}+\mu\right)} \phi_{t+T+1}(\mu) \quad \sum_{t} \phi_{n} t_{t+T+1} \mathrm{e}^{\mathrm{i}\left(n{ }^{t+T+1) \mu} D_{t}\left(\lambda_{j}+\mu\right) \text { satisfies }\right.}$ the same bounds as $\left|\phi_{n}\left(\lambda_{j}, \mu\right)\right|$ in Lemma 1 and the corollary follows.

QED

Proof of Theorem 2. We only prove again the bound for the first two expectations. We consider again first the case with $\alpha>0$. Now $E\left[w_{\zeta}\left(\lambda_{j}\right) w_{\zeta}\left(\lambda_{j}\right)\right] \quad f_{\zeta \zeta}\left(\lambda_{j}\right)$ is

$$
\begin{aligned}
E\left[I_{\zeta \zeta}\left(\lambda_{j}\right)\right] \quad f_{\zeta \zeta}\left(\lambda_{j}\right) & \frac{1}{2 \pi n} \int_{\Pi} \chi_{j, j}(\mu)\left\{f_{x x}(\mu) \quad f_{x x}\left(\lambda_{j}\right)\right\} \mathrm{d} \mu \\
& +f_{x x}\left(\lambda_{j}\right)\left\{\frac{1}{2 \pi n} \int_{\Pi} \chi_{j, j}(\mu) \mathrm{d} \mu \quad\left|\phi\left(\lambda_{j}\right)\right|^{2}\right\},
\end{aligned}
$$

where the second line is $O\left(f_{\zeta \zeta}\left(\lambda_{j}\right) j^{\alpha}{ }^{1}\right), \alpha>0$, as in Theorem 1. For the analysis of eqn (39) we consider the same intervals of integration as in Theorem 1 and only replace $f_{\eta \eta}$ by $f_{x x}$, noting that $f_{x x}(\lambda)$ is differentiable in $(0, \epsilon)$, for some $\epsilon>0$, with $f_{x x}^{\prime}(\lambda) \quad O\left(\left.|\lambda|\right|^{12 d}\right)$ as $\lambda \rightarrow 0+$. Then all bounds in Theorem 1 for eqn (19), $0<\alpha<1$, should be multiplied by $\lambda_{j}{ }^{1}$, obtaining a bound for eqn (39) of order $O\left(f_{\zeta \zeta}\left(\lambda_{j}\right) n{ }^{1} \lambda_{j}{ }^{1} \log j\right) \quad O\left(f_{\zeta \zeta}\left(\lambda_{j}\right) j{ }^{1} \log j\right)$. The only interval that requires special consideration is

$$
\begin{aligned}
\left|\int_{3 \lambda_{j} / 2}^{\lambda_{j} / 2}\right| \leq & C n^{1} \int_{3 \lambda_{j} / 2}^{\lambda_{j} / 2}\left(f_{x x}\left(\lambda_{j}\right)+f_{x x}\left(\lambda_{j}+\mu\right)\right)\left|\phi_{n}\left(\lambda_{j}, \quad \lambda_{j} \quad \mu\right)\right|^{2} \mathrm{~d} \mu \\
\leq & C n^{1} \lambda_{j}{ }^{2} \int_{0}^{\lambda_{j} / 2}|\mu|{ }^{2 \alpha} \cdot 2 d \mathrm{~d} \mu+C n^{1} \lambda_{j}{ }^{2} \int_{3 \lambda_{j} / 2}^{\lambda_{j} / 2} f_{x x}\left(\lambda_{j}\right) \lambda_{j}{ }^{2 \alpha} \mathrm{d} \mu \\
\leq & C n^{1} \lambda_{j}{ }^{2}\left|\lambda_{j}\right|^{12 \alpha .2 d}+C n^{1}\left|\lambda_{j}\right|^{2 \alpha}{ }^{2 d} \lambda_{j}{ }^{1} \\
& O\left(f_{\zeta \zeta}\left(\lambda_{j}\right) n^{1}\right) .
\end{aligned}
$$


For the second expectation considered in the theorem we can consider again the same decomposition (33) (36) replacing $f_{\eta \eta}$ by $f_{x x}$. Now proceeding as in the proof of Thm 2(c) in Robinson (1995a), considering large and small $k$, the bounds should be adapted by multiplication by $\lambda_{j}^{1}\left(\lambda_{k}^{2 d}+\lambda_{j}^{2 d}\right)$, so we obtain the bound

$$
O\left(\sqrt{ } f_{x x}\left(\lambda_{j}\right) f_{x x}\left(\lambda_{k}\right) \lambda_{k}{ }^{2 \alpha}\left(\frac{j}{k}\right)^{|d|} \lambda_{j}{ }^{1} n^{1} \log j\right) \quad O\left(\sqrt{f_{\zeta \zeta}}\left(\lambda_{j}\right) f_{\zeta \zeta}\left(\lambda_{k}\right) \frac{j^{\alpha+|d|}}{k} j^{1} \log j\right) .
$$

The only interval that requires further study is

$$
\begin{aligned}
& \frac{1}{2 \pi n} \int_{\lambda_{k} / 2}^{\lambda_{k} / 2}\left|\phi_{n}\left(\lambda_{j}, \quad \mu\right) \phi_{n}\left(\lambda_{k}, \mu\right)\right|\left\{f_{x x}(\mu) \quad f_{x x}\left(\lambda_{j}\right)\right\} \mathrm{d} \mu \\
& \leq C n{ }^{1} \lambda_{k}{ }^{1} \lambda_{j}{ }^{1} \int_{\lambda_{k} / 2}^{\lambda_{k} / 2}|\mu|^{2 d}\left(|\mu|^{\alpha}+\left|\lambda_{k}\right|^{\alpha}\right)\left(|\mu|^{\alpha}+\left|\lambda_{j}\right|^{\alpha}\right) \mathrm{d} \mu \\
& \quad+C n^{1} \lambda_{k}{ }^{1} \lambda_{j}{ }^{1} f_{x x}\left(\lambda_{j}\right) \int_{\lambda_{k} / 2}^{\lambda_{k} / 2}\left(|\mu|^{\alpha}+\left|\lambda_{k}\right|^{\alpha}\right)\left(|\mu|^{\alpha}+\left|\lambda_{j}\right|^{\alpha}\right) \mathrm{d} \mu \\
& \leq C n{ }^{1} \lambda_{k}{ }^{2 d}{ }^{2 \alpha} \lambda_{j}{ }^{1} \quad O\left(\sqrt{f_{\zeta \zeta}}\left(\lambda_{j}\right) f_{\zeta \zeta}\left(\lambda_{k}\right) \frac{j}{k}^{\alpha+|d|} j{ }^{1} \log j\right) .
\end{aligned}
$$

We now consider the case $\alpha<0$. For analysing the contribution of eqn (20) we multiply the bound for eqn (23) in Theorem 1 by $f_{x x}\left(\lambda_{j}\right)$, obtaining $O\left(f_{\zeta \zeta}\left(\lambda_{j}\right) j{ }^{1}\right)$. For the term eqn (19), we have to consider different intervals of integration of eqn (26),

$$
\begin{aligned}
\left|\int_{|\mu|<\lambda_{j} / 2}\right| & \leq C n^{1} f_{x x}^{\prime}\left(\lambda_{j}\right) \int_{|\mu|<\lambda_{j} / 2}\left|\phi_{n}\left(\lambda_{j}, \quad \lambda_{j} \mu\right)\right|^{2}|\mu| \mathrm{d} \mu \\
& \leq C n^{1} \lambda_{j}{ }^{2 \alpha} \lambda_{j}{ }^{2 d} 1 \int_{|\mu|<\lambda_{j} / 2}\left|L_{n}(\mu)\right| \mathrm{d} \mu \quad O\left(f_{\zeta \zeta}\left(\lambda_{j}\right) j^{1}\right), \\
\left|\int_{2 \lambda_{j}}{ }^{\lambda_{j} / 2}\right| & \leq C \lambda_{j}{ }^{2 \alpha^{2}} n^{1} \int_{2 \lambda_{j}}^{\lambda_{j} / 2}\left(f_{x x}\left(\lambda_{j}\right)+f_{x x}\left(\lambda_{j}+\mu\right)\right)\left|L_{n}(\mu)\right|^{2} \mathrm{~d} \mu \\
& \leq C \lambda_{j}{ }^{2 \alpha} n^{1} \lambda_{j}{ }^{2}\left(\lambda_{j}{ }^{2 d+1}+\int_{2 \lambda_{j}}^{\lambda_{j} / 2}|\mu|^{2 d} \mathrm{~d} \mu\right) \\
& \leq C \lambda_{j}{ }^{2 \alpha} n^{1} \lambda_{j}{ }^{2 d}{ }^{1} \quad O\left(f_{\zeta \zeta}\left(\lambda_{j}\right) j^{1}\right),
\end{aligned}
$$

and finally, the typical term for the remaining intervals is given by

$$
\begin{aligned}
\left|\int_{2 \lambda_{j}}^{\epsilon}\right| \leq & C n^{1} f_{x x}\left(\lambda_{j}\right) \int_{2 \lambda_{j}}^{\epsilon}\left|L_{n}(\mu)\right|^{2} \mu^{2 \alpha} \mathrm{d} \mu \\
& +C^{1}{ }^{1} \max _{2 \lambda_{j} \leq \mu \leq \epsilon} \lambda_{j}^{d}{ }^{0.5} f_{x x}\left(\lambda_{j}+\mu\right) \int_{2 \lambda_{j}}^{\epsilon}\left|L_{n}(\mu)\right|^{2} \mu^{0.5} d{ }^{2 \alpha} \mathrm{d} \mu \\
\leq & C n^{1} f_{x x}\left(\lambda_{j}\right) \lambda_{j}{ }^{12 \alpha}+C n^{1} \lambda_{j}{ }^{d} 0.5 \lambda_{j}{ }^{0.5} d d^{2 \alpha} \\
& O\left(f_{\zeta \zeta}\left(\lambda_{j}\right) j^{1}\right) .
\end{aligned}
$$

For the second expectation, first we take eqn (38) multiplied by $\sqrt{ } f_{x x}\left(\lambda_{j}\right) f_{x x}\left(\lambda_{k}\right) \quad O\left(\lambda_{j}{ }^{d} \lambda_{k}{ }^{d}\right)$. Then it is possible to estimate eqns (33) (36) for 
$0.5<\alpha<0$ using the same method of Robinson (1995a, pp. 1063 1064), because with the bound $\left|\phi_{n}\left(\lambda_{j}, \mu\right)\right| \leq C\left\{|\mu|^{\alpha}+\left|\lambda_{j}\right|^{\alpha}\right\} L_{n}\left(\lambda_{j}+\mu\right), \quad \alpha<0$, we obtain the desired result multiplying an improved bound that can be obtained collecting Robinson's results for $\alpha \quad 0$,

$$
O\left(\sqrt{f_{x x}}\left(\lambda_{j}\right) f_{x x}\left(\lambda_{k}\right)\left(\frac{j}{k}\right)^{|d|} j^{1} \log j\right) \quad O\left(\sqrt{f_{x x}}\left(\lambda_{j}\right) f_{x x}\left(\lambda_{k}\right) k^{1} \log j\right),
$$

by the factor $(j / k)^{\alpha}, \alpha<0$. We omit the details.

QED

\section{ACKNOWLEDGEMENTS}

This research was funded by the Spanish Ministerio de Educación y Ciencia (ref. no. SEJ2004 04583/ECON).

NOTE

1. This paper is a revised version of the working paper 'Log periodogram inference for nonstationary integrated and differenced processes'.

Corresponding author: Carlos Velasco, Departamento de Economía, Universidad Carlos III de Madrid, Calle Madrid 126, 28903 Getafe (Madrid), Spain. Fax: +34 91 6249875. E mail: carlos.velasco@uc3m.es.

\section{REFERENCES}

Fox, R. and TAqQU, M. S. (1986) Large-sample properties of parameter estimates for strongly dependent stationary Gaussian times series. Annals of Statistics 14, 51732.

Geweke, J. and Porter-Hudak, S. (1983) The estimation and application of long-memory time series models. Journal of Time Series Analysis 4, 22138.

Granger, C. W. J. and Joyeux, R. (1980) An introduction to long-memory time series and fractional differencing. Journal of Time Series Analysis 1, 1530.

Hassler, U., Marmol, F. and Velasco, C. (2006) Residual periodogram inference for long-run relationships. Journal of Econometrics 130, 165207.

Heyde, C. C. and YAng, Y. (1997) On defining long-range dependence. Journal of Applied Probability 34, 93944.

Hosking, J. R. M. (1981) Fractional differencing. Biometrika 68, 16576.

Hurvich, C. M. and Ray, B. K. (1995) Estimation of the memory parameter for nonstationary or noninvertible fractionally integrated processes. Journal of Time Series Analysis 16, 1742.

Hurvich, C. M., Deo, R. and Brodsky, J. (1998) The mean square error of Geweke and PorterHudak's estimator of the memory parameter of a long-memory time series. Journal of Time Series Analysis 19, 1946.

Hurvich, C. M., Moulines, E. and Soulier, P. (2002) The FEXP estimator for potentially non-stationary linear time series. Stochastic Processes and their Applications 97, 30740.

Kim, C. S. and Phillips, P. C. B. (1999) Log-periodogram Regression: The Nonstationary Case. Preprint, New Haven, CT: Cowles Foundation for Research in Economics, Yale University. 
KüNSCH, H. R. (1987) Statistical aspects of self-similar processes. In Proceedings 1st World Congress of the Bernoulli Society, Utrecht: VNU Science Press, pp. 67-74.

Marinucci, D. and Robinson, P. M. (2000) Weak convergence of multivariate fractional processes. Stochastic Processes and their Applications 86, 103-20.

Marmol, F. and Velasco, C. (2001) Trend stationarity versus long range dependence in time series analysis. Journal of Econometrics 108, 25-42.

Marmol, F. and Velasco, C. (2004) Consistent testing of fractional cointegration. Econometrica 72, 1809-44.

Phillips, P. C. B. (1999) Discrete Fourier transforms of fractional processes. Cowles Foundation for Research in Economics Discussion Paper 1243, Yale University.

Phillips, P. C. B. and Solo, V. (1992) Asymptotics for linear processes. Annals of Statistics 20, 9711001.

Robinson, P. M. (1995a) Log-periodogram regression of time series with long range dependence. Annals of Statistics 23, 1048-72.

Robinson, P. M. (1995b) Gaussian semiparametric estimation of long range dependence. Annals of Statistics 23, 1630-61.

Robinson, P. M. (2005) The distance between rival nonstationary processes. Journal of Econometrics $128,283-300$.

Robinson, P. M. and MarinucCi, D. (2001) Narrow-band analysis of nonstationary processes. Annals of Statistics 29, 947-86.

Solo, V. (1992) Intrinsic random functions and the paradox of 1/f noise. Siam Journal of Applied Mathematics 52, 270-91.

Sowell, F. B. (1990) The fractional unit root distribution. Econometrica 58, 495-04.

Velasco, C. (1999) Non-stationary log-periodogram regression. Journal of Econometrics 91, 325-71.

Velasco, C. (2000) Non-Gaussian log-periodogram regression. Econometric Theory 16, 44-79.

Velasco, C. (2006) Semiparametric estimation of long-memory models. In Palgrave Handbook of Econometrics, Vol. 1. Econometric Theory (eds K. Patterson and T. C. Mills). Basingstoke: Palgrave Macmillan, pp. 353-395.

Velasco, C. and Robinson, P. M. (2000) Whittle pseudo-maximum likelihood estimates of nonstationary time series. Journal of the American Statistical Association 95, 1229-43. 